\title{
Sind leistungsschwache Jugendliche tatsächlich nicht ausbildungsfähig?
}

\author{
Eine Längsschnittanalyse zur beruflichen \\ Qualifizierung von Jugendlichen mit geringen \\ kognitiven Kompetenzen im Nachbarland Schweiz
}

\author{
Sandra Buchholz • Christian Imdorf • Sandra Hupka-Brunner • \\ Hans-Peter Blossfeld
}

Zusammenfassung: In den vergangenen Jahren wurde in Deutschland immer wieder die mangelnde Ausbildungsreife von Jugendlichen beklagt. Demnach soll es einen relativ hohen Prozentsatz von Jugendlichen geben, deren kognitive Kompetenzen so gering sind, dass sie nicht erfolgreich in die berufliche Ausbildung integriert werden können. Ziel des vorliegenden Beitrages ist es, die Ausbildungsfähigkeit von Jugendlichen mit geringen kognitiven Kompetenzen im Längsschnitt zu untersuchen und zwar für das Nachbarland Schweiz, dessen Ausbildungssystem dem Deutschlands in vielen Aspekten ähnelt und das in PISA einen vergleichbar hohen Anteil von Schülern mit geringen kognitiven Kompetenzen aufweist. Dazu werden wir auf Daten der schweizerischen TREE-Studie zurückgreifen, mit welcher wir Jugendliche mit geringen Kompetenzen identifizieren und deren (Ausbildungs-) Übergänge nach Ende der Schulpflicht nachzeichnen können. Wie unsere Ergebnisse zeigen, lassen sich in der Schweiz auch Jugendliche mit geringeren kognitiven Kompetenzen erfolgreich in den beruflichen Ausbildungsmarkt integrieren. Anders als Deutschland ist die Schweiz somit deutlich erfolgreicher, auch schwächeren Jugendlichen eine Ausbildungschance zu bieten.

Schlüsselwörter: Berufliche Ausbildung · Ausbildungsreife $\cdot$ Leistungsschwache Jugendliche · Schweiz $\cdot$ Deutschland

(C) Springer Fachmedien Wiesbaden 2012

S. Buchholz $(\bowtie)$

Lehrstuhl für Soziologie 1, Otto-Friedrich-Universität Bamberg,

96045 Bamberg, Deutschland

E-Mail: sandra.buchholz@uni-bamberg.de

Ch. Imdorf · S. Hupka-Brunner

Institut für Soziologie, Universität Basel,

Petersgraben 27, 4051 Basel, Schweiz

E-Mail: christian.imdorf@unibas.ch

S. Hupka-Brunner

E-Mail: sandra.hupka@unibas.ch

H.-P. Blossfeld

Department of Political and Social Sciences, European University Institute,

Via dei Roccetini 9, 50014 San Domenico di Fiesole, Italien

E-Mail: hp.blossfeld@eui.ei 


\title{
Are academically weak youths really not ready for vocational training? $-\mathbf{A}$ longitudinal analysis of vocational training trajectories of youths with low cognitive competencies in Switzerland
}

\begin{abstract}
For several years, there is an increasing discussion in Germany that youths are less and less ready for vocational training. According to these reports that have been published by employers' representatives, there exists a relatively high share of youths whose cognitive competencies are too low to be successfully integrated into the German dual training system. Our article aims at investigating the maturity of youths with low cognitive competencies in a longitudinal design for Switzerland. The Swiss educational system resembles the German educational system in various main aspects and PISA has shown that the share of pupils with low competencies is comparably high in both countries. For our empirical analyses, we will use youth panel data from the Swiss TREE study which allows us to identify youths with low cognitive competencies and to follow their training trajectories after finishing compulsory schooling. Our results show that in the German neighbour Switzerland also youths with low competencies can be successfully integreated into the vocational training system. In contrast to Germany, the Swiss system thus is far more successful in offering also academically weak youths to obtain vocational training.
\end{abstract}

Keywords: Vocational training $\cdot$ Readiness for training $\cdot$ Academically weak youths · Switzerland · Germany

\section{Einleitung: Das deutsche Berufsausbildungssystem - vom Erfolgsmodell zum System mit Integrationsproblemen für leistungsschwache Jugendliche}

Das berufliche Ausbildungssystem Deutschlands gilt als Erfolgsmodell, welches im internationalen Vergleich als besonders leistungsfähig wahrgenommen und dessen Beitrag zur Integration nachwachsender Generationen in den Arbeitsmarkt als weit überdurchschnittlich bewertet wird (vgl. z. B. Blossfeld und Stockmann 1998/1999; Scherer 2005; Brzynski-Fay 2007). Insbesondere das duale Ausbildungssystem ist das Aushängeschild Deutschlands im Ausland. In der Tat zeigt sich, dass kein in anderen modernen Ländern existierendes Berufsausbildungssystem, sei es schulisch oder auf „on-the-job training“ basierend, auch nur annähernd so erfolgreich ist, Jugendarbeitslosigkeit zu reduzieren und nachwachsende Generationen über einen qualifizierten Berufsabschluss adäquat in den Arbeitsmarkt zu integrieren (vgl. z. B. Müller und Shavit 1998; Scherer 2005; Brzinsky-Fay 2007).

Trotz dieser insgesamt positiven Bewertung zeigte sich jedoch bereits seit mehreren Jahren, dass das deutsche Berufsausbildungssystem in seiner jetzigen Form zunehmend an seine Grenzen gestoßen ist. Vor allem gelingt es seit einigen Jahren immer weniger, die Nachfrage von Jugendlichen nach einer voll berufsqualifizierenden Ausbildung erfolgreich abzudecken. Derzeit entfallen ein Drittel (und mehr) der Neuzugänge im deutschen Ausbildungssystem auf das sogenannte Übergangssystem (z. B. Ulrich 2008; Autorengruppe Bildungsberichterstattung 2006, 2008, 2010). ${ }^{1}$ Das Übergangssystem fängt in

1 Wir bitten zu beachten, dass diese und die im Folgenden von uns für Deutschland berichteten Werte nicht auf Jahrgänge oder Abschlusskohorten basieren sondern Neuzugänge zum beruflichen Ausbildungssystem in einem bestimmten Jahr darstellen. Somit sind diese Werte nicht 
Deutschland diejenigen Jugendlichen auf, denen es nicht gelungen ist, einen regulären und voll qualifizierenden Ausbildungsplatz im Schulberufs- oder dualen Ausbildungssystem zu finden. Maßnahmen im Übergangssystem haben zum Ziel, diesen Jugendlichen verbesserte Basisqualifikationen zu vermitteln und damit ihre zukünftigen Chancen auf einen Ausbildungsplatz zu erhöhen. Es handelt sich hierbei also um „(Aus-) Bildungsangebote, die unterhalb einer qualifizierten Berufsausbildung liegen oder zu keinem anerkannten Ausbildungsabschluss führen, sondern auf eine Verbesserung der individuellen Kompetenzen von Jugendlichen zur Aufnahme einer Ausbildung oder Beschäftigung zielen und zum Teil das Nachholen eines allgemeinbildenden Schulabschlusses ermöglichen“ (Autorengruppe Bildungsberichterstattung 2006, S. 79).

Für das starke Anwachsen des Übergangssystems in den vergangenen Jahren werden gerade von Arbeitgeberseite geringe kognitive Kompetenzen und somit eine mangelnde Ausbildungsfähigkeit von Jugendlichen verantwortlich gemacht (vgl. Eberhard 2006). So veröffentlichte die Deutsche Industrie- und Handelskammer (DIHK) im Jahr 2009 eine Expertise, in der $64 \%$ der befragten Unternehmen angaben, ihre offenen Lehrstellen nicht besetzen zu können, weil die Bewerber über mangelnde Fähigkeiten verfügten und somit für eine berufliche Ausbildung ungeeignet seien. Entsprechend ist es nicht überraschend, dass sich insbesondere schulisch schwache Jugendliche in Deutschland nach Verlassen des allgemeinbildenden Schulsystems sehr häufig im Übergangssystem wiederfinden (Baethge 2008). So entfielen im Jahr 2008 nur knapp 23 \% der Neuzugänge von Jugendlichen ohne Hauptschulabschluss auf das Schulberufssystem oder das duale Ausbildungssystem, während mehr als drei Viertel der Neuzugänge in das Übergangssystem erfolgten (Autorengruppe Bildungsberichterstattung 2010). Aber auch unter Jugendlichen mit Hauptschulabschluss ist der Anteil der Neuzugänge ins Übergangssystem mit knapp über $50 \%$ noch beträchtlich hoch; unter Jugendlichen mit Realschulabschluss sind es immerhin noch $18,4 \%$ (ebd.).

Insgesamt scheint der Erfolg dieser Übergangsmaßnahmen jedoch eher fraglich (Baethge et al. 2007; Baethge 2008; Gaupp und Reißig 2006; Reißig et al. 2008). Denn nur einem kleineren Teil der Jugendlichen gelingt nach Teilnahme an solchen berufsvorbereitenden Maßnahmen der Übergang in ein reguläres, voll qualifizierendes Ausbildungsverhältnis (Baethge 2008). Insbesondere für Jugendliche mit und ohne Hauptschulabschluss ist das Übergangssystem oftmals eine Sackgasse (Gaupp et al. 2008). Somit versagt das deutsche Berufsausbildungssystem zunehmend darin, leistungsschwachen Mitgliedern der nachwachsenden Generationen einen Zugang zu Ausbildungsplätzen zu geben, die ihnen einen berufsbefähigenden Abschluss oder zumindest die Aussicht auf einen solchen bieten. Dabei ist gerade mit Blick auf den durch den demografischen Wandel ausgelösten drohenden Fachkräftemangel dieses systematische Brachlegen von Humankapitalressourcen in Deutschland höchst problematisch. Künftig wird die Zahl der Schulabgänger, die eine Ausbildung antreten können, stark schrumpfen (Bundesministerium für Bildung

direkt mit denen in unseren empirischen Analysen für die Schweiz vergleichbar, da unsere schweizerischen Analysen eine Abschlusskohorte in den Blick nehmen. Miteinander vergleichbare Zahlen für die Bedeutung des dualen Ausbildungssystems, des Schulberufssystems und des Übergangssystems in Deutschland und der Schweiz zeigen, dass Übergangsmaßnahmen in der Schweiz eine weit geringere Bedeutung haben (vgl Seibert et al. 2009). 
und Forschung 2008). Das deutsche Berufsausbildungssystem muss sich also darauf vorbereiten, die Humankapitalressourcen für den Arbeitsmarkt und die Wirtschaft nachhaltiger und effizienter zu sichern; auch durch Rückgriff auf Jugendliche, denen bisher eine mangelnde Ausbildungsreife zugeschrieben wird.

Ziel unseres Beitrages ist es, die Ausbildungschancen von leistungsschwachen Jugendlichen nach Ende der Schulpflicht zu untersuchen. Konkret werden wir die beruflichen Ausbildungschancen von Jugendlichen mit einer geringen kognitiven Kompetenzstufe in PISA 2000 analysieren und diese mit den Chancen von Jugendlichen mit mittleren und hohen kognitiven Kompetenzen vergleichen. Wichtig ist es darauf hinzuweisen, dass wir uns somit auf die kognitiven Fähigkeiten von Jugendlichen als Indikator für Ausbildungsreife konzentrieren (für eine detaillierte Ausführungen und Begründung hierzu siehe Abschn.3). Für unsere Analysen werden wir auf Daten aus dem Nachbarland Schweiz zurückgreifen, nämlich auf die Daten der aus der PISA-Erhebung 2000 entstandenen Längsschnittstudie „Transitions from Education to Employment“ (TREE). Mit den TREE-Daten können wir, anders als für Deutschland, wo solche Daten bisher nicht zur Verfügung stehen, Jugendliche mit niedrigem kognitivem Kompetenzniveau identifizieren und deren Ausbildungsverlauf nachvollziehen. Ob diese Jugendlichen tatsächlich nicht ausbildungsfähig sind, werden wir für die Schweiz, dessen Ausbildungssystem dem Deutschlands in vielen Aspekten ähnlich ist und das in PISA einen vergleichbar hohen Anteil von Schülern mit geringen kognitiven Kompetenzen aufweist (siehe nachfolgender Abschn.2), im Längsschnitt unter Verwendung ereignisanalytischer Modelle untersuchen.

\section{Formale Ähnlichkeiten des (Aus-) Bildungssystems und strukturelle Unterschiede am Ausbildungsmarkt: Die Schweiz und Deutschland im Vergleich}

Grundsätzlich ähneln sich die Bildungssysteme der Schweiz und Deutschlands in vielen Aspekten. ${ }^{2}$ In beiden Ländern beträgt die obligatorische Schulzeit 9 oder 10 Jahre, beide Schulsysteme zeichnen sich durch eine starke und frühe Aufteilung von Schülern auf unterschiedliche Schullaufbahnen aus (das sogenannte „Tracking“), auch sind beide Systeme föderal organisiert. Zwar gibt es in der Schweiz anders als in Deutschland keine formalen Sekundarschulabschlüsse, jedoch lassen sich trotz kantonaler Unterschiede auch in der Schweiz auf Niveau der Sekundarstufe I überkantonal drei Schultypen unterscheiden: 1) Schulen mit Grundanforderungen (traditionell in der Schweiz als „Realschulen“ bezeichnet), 2) Schulen mit erweiterten Anforderungen und 3) Schulen mit hohen Anforderungen. Dabei entsprechen Schulen mit Grundanforderungen in der Schweiz in etwa den Hauptschulen in Deutschland (Hupka-Brunner et al. 2011), mit dem Unterschied, dass den schweizerischen Schulen mit Grundanforderungen weniger das für die deutsche Hauptschule verbreitete Stigma einer „Restschule“ anhaftet. Schulen mit hohen Anforderungen sind vergleichbar mit den deutschen Gymnasien. Bei den Schulen mit erweiterten

2 Einen detaillierten Überblick über das schweizerische Bildungssystem geben beispielsweise Bundesamt für Statistik (2008), Stalder und Nägele (2011) und Schweizerische Koordinationsstelle für Bildungsforschung (2010). 
Anforderungen gibt es größere Unterschiede zwischen den Kantonen. Beispielsweise entspricht dieser Schultyp im zweigliedrigen Schulsystem des Kantons Bern dem höchsten Sekundarschulniveau. In Kantonen mit dreigliedrigen Schulsystemen sind es dagegen Sekundarschulen im mittleren Leistungssegment. Diese insgesamt großen Ähnlichkeiten zwischen dem deutschen und schweizerischen Schulsystem gehen nicht überraschend einher mit einer vergleichbaren Leistungsstreuung der Schülerinnen und Schüler. So hat PISA gezeigt, dass in beiden Ländern der Anteil von Schülern mit geringen und hohen Lesekompetenzen sehr ähnlich ist (Artelt et al. 2002). Auch im mittleren Kompetenzbereich schneidet die Schweiz sehr ähnlich wie Deutschland ab (Bundesamt für Statistik und Schweizerische Konferenz der kantonalen Erziehungsdirektoren 2002, S. 31).

Aber nicht nur mit Blick auf das allgemeinbildende Schulsystem sondern auch mit Blick auf das berufliche Ausbildungssystem ähneln sich Deutschland und die Schweiz. In beiden Ländern dominiert das duale System, welches eine theoretische Ausbildung in Berufsschulen mit einer praktischen Qualifizierung in einem Ausbildungsbetrieb verbindet. Bezeichnend ist am dualen System im Vergleich zu anderen Ausbildungssystemen, zum einem dem nicht formalisierten „on the job training“, zum anderen der rein schulisch organisierten beruflichen Ausbildung, dass erfolgreiche Absolventen sowohl praktische Erfahrungen im Unternehmen gesammelt haben, gleichzeitig aber auch über ein standardisiertes und anerkanntes Berufszertifikat verfügen, welches ihnen erlaubt, flexibel (innerhalb des erlernten Berufes) zwischen Unternehmen wechseln zu können (Müller und Shavit 1998). Im Vergleich zur Organisation beruflicher Ausbildung in anderen Ländern wird das duale Ausbildungssystem deshalb als ein System bewertet, das nachwachsenden Generationen einen relativ reibungslosen Übergang in den Arbeitsmarkt ermöglicht und zudem sehr gut ausgebildete Fachkräfte für den Arbeitsmarkt zur Verfügung stellt (vgl. z. B. Blossfeld und Stockmann 1998/1999; Müller et al. 1998, 2002). Die Schattenseite des dualen Systems ist jedoch, dass vor allem Unqualifizierte und bildungsschwache Personen Gefahr laufen, aufgrund eines fehlenden Zertifikats keinen Einstieg in qualifizierte Arbeit zu finden und dann dauerhaft aus dem primären Arbeitsmarkt ausgeschlossen zu sein. Diese Gefahr ist dann besonders groß, wenn die Konkurrenz um die beruflichen Ausbildungsplätze hoch ist. Das gänzliche Fehlen eines Berufszertifikats stellt auf Arbeitsmärkten mit dualem Ausbildungssystem für den späteren Erwerbsverlauf eine kaum überwindbare Hürde dar. Der Zugang zu einer dualen Ausbildung ist somit von zentraler Bedeutung für die langfristigen Arbeitsmarktchancen der jungen Generationen in diesen Ländern.

Mit Blick auf das Angebot für Jugendliche, denen ein Übergang in eine Regellehre im dualen Ausbildungssystem nicht gelingt, gibt es zwischen Deutschland und der Schweiz sowohl Ähnlichkeiten als auch Unterschiede. In Deutschland finden sich diese Jugendlichen im sogenannten Übergangssystem wieder. Es handelt sich um ein System, in welchem ein sehr heterogenes und wenig standardisiertes Angebot zusammengefasst wird. Wie eingangs ausgeführt, hat die Bedeutung des Übergangssystems am deutschen Ausbildungsmarkt in den 2000er Jahren deutlich zugenommen, um, im Unterschied zur Schweiz (vgl. Seibert et al. 2009), den Abbau von betrieblichen Ausbildungsplätzen im dualen System zu kompensieren. Insgesamt ist das Angebot im deutschen Übergangssystem sehr unübersichtlich und es existieren die verschiedensten Träger, Zuständigkeiten und Ziele. Den größten Anteil im deutschen Übergangssystem machen die ein- und 
zweijährigen Berufsfachschulen aus (2010: 36\%), die Teilnehmern keinen beruflichen Abschluss vermitteln (Autorengruppe Bildungsberichterstattung 2010). An einigen dieser Berufsfachschulen können Schüler neben dem Erwerb beruflicher Grundkenntnisse auch allgemeinbildende Abschlüsse (wie Realschulabschlüsse oder die Fachschulreife) nachholen. Trotz einem Rückgang seit 2006 stellen berufsvorbereitende Maßnahmen der Bundesagentur für Arbeit den zweitgrößten Anteil im deutschen Übergangssystem (2010: $20 \%$; Autorengruppe Bildungsberichterstattung 2010). Das Ziel berufsvorbereitender Maßnahmen der Bundesagentur für Arbeit ist es, die individuellen Voraussetzungen der teilnehmenden Jugendlichen für die Aufnahme einer Berufsausbildung zu verbessern. Der danach noch verbleibende Anteil am Übergangssystem besteht vor allem aus Berufsschülern ohne Ausbildungsvertrag (2010: 15\%), dem Berufsvorbereitungsjahr (2010: 13\%) und dem schulischen Berufsgrundbildungsjahr (2010: 11\%) (Autorengruppe Bildungsberichterstattung 2010). Die verbleibenden knapp 5\% setzten sich 2010 aus Einstiegsqualifizierungen und, mit unter $1 \%$ Anteil, Praktika vor Erzieherausbildungen zusammen (Autorengruppe Bildungsberichterstattung 2010).

Auch in der Schweiz existieren vergleichbare Programme für Jugendliche, denen ein Übergang in eine reguläre Ausbildung nicht gelungen ist. In der Schweiz werden diese Programme unter dem Begriff „Brückenangebote“ zusammengefasst. Wie auch in Deutschland handelt es sich hier um sehr unterschiedliche Programme, die zum Ziel haben, die schulischen Kompetenzen und die Berufswahlorientierung der Jugendlichen zu fördern. Das Absolvieren eines freiwilligen 10. Schuljahres, um etwaige schulische Defizite auszugleichen, ist innerhalb des schweizerischen Überbrückungssystems besonders verbreitet. Neben diesem schulischen Angebot existiert im schweizerischen Überbrückungssystem die sogenannte Vorlehre. Dabei handelte es sich um ein einjähriges Berufsvorbereitungsjahr, das dual, also in einem Betrieb und an einer Berufsschule, absolviert wird und prinzipiell in allen Berufen möglich ist. Darüber hinaus existieren weitere Angebote wie Motivationssemester (durch die Arbeitslosenversicherung finanzierte Programme für stellenlose Jugendliche ohne abgeschlossene berufliche Grundbildung) und Vorkurse (für gestalterische Berufe und Gesundheitsberufe) sowie weniger institutionalisierte Überbrückungsmaßnahmen wie Praktika und Sprachaufenthalte (etwa die nach wie vor verbreiteten Au-pair-Aufenthalte von Deutschschweizer Mädchen in der französischsprachigen Schweiz), welche von Jugendlichen als Zwischenlösungen genutzt werden. Im Unterschied zu Deutschland dienen die Überbrückungsmaßnahmen in der Schweiz jedoch kaum der Teil- und Nachqualifizierung, da die Schweiz keine Schulabschlüsse kennt.

Trotz dieser insgesamt großen Ähnlichkeiten des schweizerischen und deutschen Schul- und (Aus-) Bildungssystems zeigen sich dennoch gleichzeitig wichtige strukturelle Unterschiede am Ausbildungsmarkt beider Länder, welche insbesondere für die hier gestellte Frage zu den Ausbildungschancen von Jugendlichen mit geringen Kompetenzen bedeutend sein dürften.

Der erste und wohl augenscheinlichste Unterschied liegt in der sehr unterschiedlichen Arbeitsmarktlage der beiden Länder. Die allgemeine Arbeitsmarkt- und damit auch die Ausbildungsmarktlage ist in der Schweiz, auch trotz eines Anstiegs der Arbeitslosenquote in der jüngeren Vergangenheit, deutlich entspannter als in Deutschland (siehe Abb.1). Dies gilt insbesondere für die Periode, in welcher die von uns im Folgenden mit den 


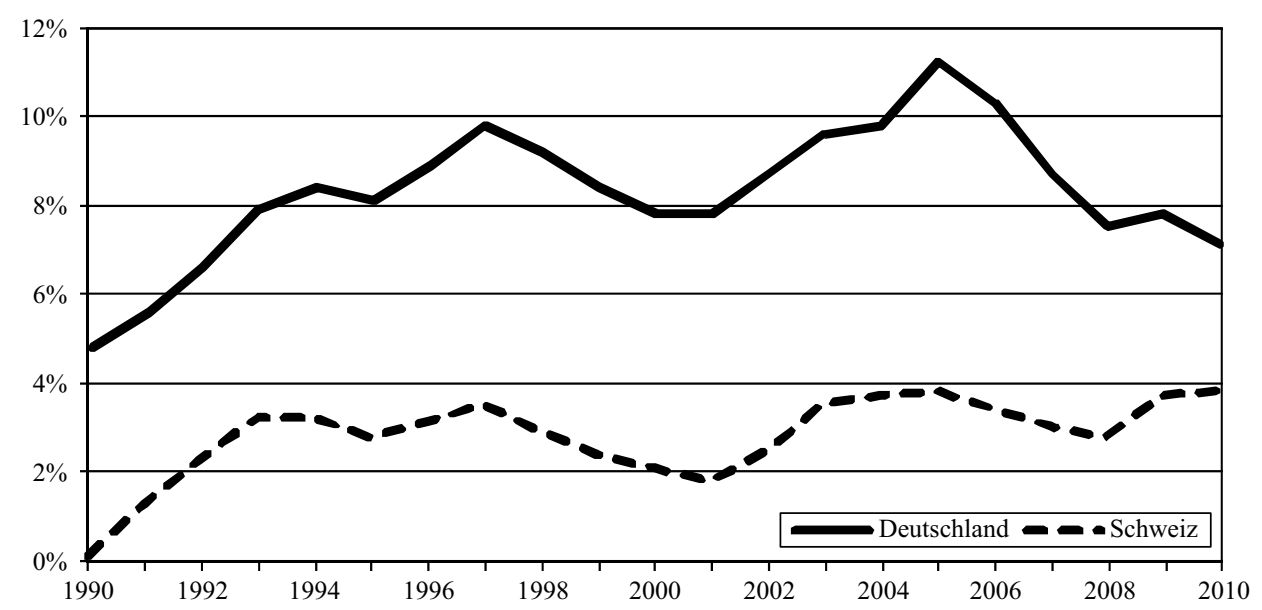

Abb. 1: Arbeitslosenquote in Deutschland und der Schweiz (1990 bis 2010). (Quelle: OECD Labor Force Statistics)

TREE-Daten untersuchten schweizerischen Jugendlichen die Schulpflicht beendet haben und erstmals in eine berufliche Ausbildung übergehen konnten (d.h. in den Jahren 2000 bis 2002). In diesen Jahren war die Arbeitslosenquote in der Schweiz mit einem Wert von maximal 3\% äußerst niedrig, und der schweizerische Arbeitsmarkt hatte mehrere Jahre eines kontinuierlichen Aufschwungs sowie eine Halbierung der Arbeitslosenquote erlebt.

Entsprechend der mikro-ökonomischen Arbeitsmarkttheorie (Sørensen und Kalleberg 1981; Thurow 1979) müsste sich diese gute Arbeitsmarktlage auch positiv auf die Ausbildungschancen von leistungsschwachen Jugendlichen in der Schweiz auswirken. Demnach werden Bewerber bei der Besetzung von Ausbildungsplätzen (wie auch Arbeitsstellen allgemein) durch Arbeitgeber entsprechend ihrer anzunehmenden Produktivität gereiht, für deren Beurteilung verschiedene Leistungsindikatoren stellvertretend herangezogen werden. Leistungsschwache Jugendliche würden in dieser Reihung folglich auf hintere Plätze verwiesen werden, da sie als schwächer eingestuft werden als leistungsstärkere Jugendliche. In einer angespannten Arbeitsmarktlage mit einem Überschuss an Bewerbern wie in Deutschland, werden leistungsschwache Jugendliche dann schlichtweg von den als produktiver eingeschätzten Mitbewerbern verdrängt (vgl. auch Blossfeld 1985). Die gute Arbeitsmarktlage in der Schweiz dürfte dagegen die Chancen von leistungsschwachen Jugendlichen deutlich verbessern. Das geringe Leistungsniveau von Jugendlichen wäre entsprechend dieser Argumentation also kein absolutes sondern ein relatives Ausschlusskriterium am Ausbildungsmarkt.

Der zweite wichtige Unterschied zwischen Deutschland und der Schweiz, der sich deutlich auf die Chancen leistungsschwacher Jugendlicher auswirken dürfte, liegt in der sehr unterschiedlichen Größenstruktur der Ausbildungsbetriebe in den beiden Ländern (bei vergleichbarer Verteilung der Ausbildungsplätze auf den zweiten und dritten Wirtschaftssektor; siehe Müller und Schweri 2012, S. 70; BIBB 2012). Anders als in Deutschland findet in der Schweiz die Ausbildung viel häufiger durch Mikro- und Kleinbetriebe mit maximal 49 Mitarbeitern statt (siehe Tab. 1). So wird in Deutschland nur knapp die 
Tab. 1: Lehrstellenangebot nach Betriebsgröße (in Prozent) - Deutschland und die Schweiz im Vergleich

\begin{tabular}{lccccc}
\hline & \multicolumn{2}{c}{ Deutschland $^{\mathrm{a}}$} & & \multicolumn{2}{l}{ Schweiz $^{\mathrm{b}}$} \\
\cline { 2 - 3 } \cline { 5 - 6 } & 1999 & 2007 & & 2001 & 2005 \\
\hline Betriebsgröße & & & & & 31 \\
Mikro (1 bis 9 Mitarbeiter) & 23 & 22 & & 31 & 34 \\
Klein (10 bis 49 Mitarbeiter) & 27 & 25 & & 35 & 34 \\
Mittel (50 bis 249 Mitarbeiter) & 23 & 25 & & 20 & 20 \\
Groß (250 und mehr Mitarbeiter) & 28 & 28 & & 14 & 12 \\
Gesamt & 100 & 100 & & 100 & 100 \\
\hline
\end{tabular}

${ }^{a}$ Eigene Berechnungen mit Daten der Bundesagentur für Arbeit (Beschäftigtenstatistik, vgl. BIBB 2010)

${ }^{b}$ Eigene Berechnungen mit Daten des Bundesamts für Statistik (Betriebszählungen 2001 und 2005, vgl. BFS 2008)

Hälfte der Ausbildungen in Mikro- und Kleinbetrieben absolviert (1999: 50\%, 2007: $47 \%$ ). In der Schweiz erfolgt dagegen ein weitaus größerer Teil der Ausbildungen, nämlich zwei Drittel, in Mikro- und Kleinbetrieben (2001: 66\%, 2007: 68\%). Weiterhin ist auffällig, dass in Deutschland gerade große Betriebe mit 250 und mehr Mitarbeitern deutlich stärker am Ausbildungsvolumen beteiligt sind als in der Schweiz. In Deutschland werden $28 \%$ der Ausbildungen in großen Betrieben absolviert, in der Schweiz hingegen nur $12-14 \%$.

Dieser Unterschied in der Größenstruktur der am Ausbildungsmarkt beteiligten Betriebe ist für die von uns hier gestellte Frage insofern wichtig, als davon auszugehen ist, dass die Auswahlverfahren für die Besetzung von Ausbildungsplätzen in kleinen und großen Betrieben sehr verschieden sind. Insbesondere von größeren Unternehmen ist bekannt, dass schulische Leistungen bei der Vergabe von Ausbildungsplätzen stärker beachtet werden als in kleineren Betrieben (Seibert et al. 2009). Im Gegensatz zu Kleinunternehmen hat in Großbetrieben die schulische Leistung von Bewerbern bei der Vorselektion von Auszubildenden eine große Bedeutung (Moser 2004): zum einen, da sie aufgrund des stärkeren Bewerberandrangs auf kostengünstige Formen der frühen Auslese angewiesen sind (Imdorf 2009); zum anderen, da die Ausbildungsnettokosten in Großbetrieben höher sind als in kleinen Betrieben (vgl. Mühlemann et al. 2007, S. 73) und größere Firmen deshalb das Risiko potenzieller Ausbildungsfehlinvestitionen zu vermeiden versuchen, indem sie bei der Auswahl von Auszubildenden von Personen mit unterdurchschnittlichen Schulleistungen absehen (Imdorf 2009). Das stärkere Engagement von Mikro- und Kleinbetrieben am Lehrstellenangebot in der Schweiz könnte somit zusätzlich dazu beitragen, dass die Ausbildungschancen Jugendlicher mit geringen Kompetenzen dort vergleichsweise gut sind.

\section{Fragestellung, Forschungsdesign und Arbeitshypothesen}

Die zentrale Frage, die uns in den folgenden empirischen Analysen interessiert, ist, ob leistungsschwache Jugendliche tatsächlich nicht ausbildungsfähig sind. Konkret werden 
wir dazu in der Schweiz den beruflichen Ausbildungsverlauf nach Ende der Schulpflicht von Jugendlichen mit einer geringen Lesekompetenzen in PISA 2000 mit denen von Jugendlichen mit mittleren und hohen Lesekompetenzen vergleichen. Für Deutschland liegen bisher leider keine Längsschnittdaten vor, die uns erlauben, (Aus-) Bildungsübergänge von Jugendlichen mit unterschiedlichem Kompetenzniveau nach Ende der Schulpflicht vergleichend zu analysieren. Für das Nachbarland Schweiz gibt es mit der aus der 2000er PISA-Untersuchung hervorgegangenen Studie „Transitions from Education to Employment" (TREE) aber solche Längsschnittdaten.

PISA hat gezeigt, dass der Anteil von Jugendlichen mit geringen Kompetenzen in Deutschland und der Schweiz ähnlich ist. Zudem ähnelt das schweizerische (Aus-) Bildungssystem dem Deutschlands formal in vielen Punkten: So finden wir auch in der Schweiz ein eher stratifiziertes Schulsystem, das Jugendliche schon vergleichsweise früh in verschiedene Laufbahnen einteilt. Wie in Deutschland dominiert auch in der Schweiz die berufliche Ausbildung in einem dualen System. Und auch mit Blick auf den institutionellen Umgang mit Jugendlichen, denen zunächst die Aufnahme einer regulären Lehre nicht gelingt, ähneln sich beide Länder. Wegen dieser großen Ähnlichkeiten ist die Schweiz ein spannender Testfall für die in Deutschland häufig geäußerte These der mangelnden Ausbildungsreife oder Ausbildungsfähigkeit von kompetenzschwachen Jugendlichen. Denn anders als in Deutschland ist die schweizerische Arbeitsmarktlage weit entspannter, was für die Ausbildungschancen leistungsschwacher Jugendlicher ebenso von Vorteil sein dürfte wie die stärkere Beteiligung von Klein- und Mikrobetrieben am schweizerischen Ausbildungsmarkt.

Um die Integrationsfähigkeit von kompetenzschwachen Jugendlichen in eine berufliche Ausbildung zu untersuchen, werden wir in den nachfolgenden Analysen folgende Übergänge betrachten: Im ersten Schritt nehmen wir Ausbildungsübergänge von leistungsschwachen und leistungsstarken Jugendlichen nach Ende der Schulpflicht in den Blick. Dazu unterscheiden wir, ob diese Jugendlichen 1) eine berufliche Ausbildung aufnehmen, 2) lediglich einen Übergang in sogenannte Überbrückungsmaßnahmen schaffen oder 3) eine schulische Allgemeinbildung der Sekundarstufe II antreten. Im zweiten Schritt nehmen wir das intellektuelle Anforderungsniveau der aufgenommenen beruflichen Ausbildung in den Blick und unterscheiden zwischen beruflichen Ausbildungen mit 1) geringen ${ }^{3}, 2$ ) mittleren und 3 ) hohen Anforderungen. So wollen wir die Frage klären, ob die Integration von leistungsschwachen Jugendlichen vor allem durch das Aufnehmen von Ausbildungen mit einem geringen intellektuellen Anforderungsniveau zu erklären ist.

Es ist wichtig darauf hinzuweisen, dass wir uns in unseren Analysen auf die kognitiven Fähigkeiten als Indikator für die Ausbildungsreife von Jugendlichen konzentrieren. Bisher liegt auch von wissenschaftlicher Seite kein einheitliches und trennscharfes Konzept für Ausbildungsreife vor (Dietrich et al. 2009; Eberhard 2006). Bei einer genaueren

3 In diesem Anforderungsniveau enthalten sind auch die Anlehre und zweijährige berufliche Grundausbildung mit Attest, die im Jahr 2003 eingeführt wurde und die Anlehre als Ausbildungsform ablöste. Diese stellt ein spezielles Berufsbildungsangebot für Abgängerinnen und Abgänger insbesondere aus Sonderschulen und Sonderklassen dar und qualifiziert schulleistungsschwache Jugendliche so, dass ihre Arbeitsmarktchancen gewährleistet sind (Kammermann und Hättich 2010). 
Betrachtung des Diskurses um Ausbildungsreife zeigt sich sogar, dass die verschiedenen am Ausbildungsmarkt beteiligten Akteure sehr unterschiedliche Merkmale benennen, um Ausbildungsreife zu charakterisieren (Eberhard 2006). Dies gilt insbesondere, wenn man die Aussagen von Arbeitgeber- und Arbeitnehmervertretern miteinander vergleicht. Eberhard (2006, S. 56) weist darauf hin, dass dieser Unterschied vor allem auf die verschiedenen Interessen und Legitimationsversuche dieser Akteure zurückzuführen ist und ,vor dem Hintergrund der Lehrstellenproblematik [...] beide Seiten dazu [neigen], das eigene Verhalten situativ, das Verhalten der Gegenseite personenbezogen zu begründen“. So machen Gewerkschaftsvertreter vor allem eine gesunkene Ausbildungsverantwortung der Wirtschaft für die Diskussion um mangelnde Ausbildungsreife verantwortlich, während Wirtschaftsvertreter eine tatsächliche Verschlechterung von Kompetenzen und eine mangelnde Eignung von Bewerbern feststellen. In dem 2009 veröffentlichten Kriterienkatalog des „Nationalen Paktes für Ausbildung und Fachkräftenachwuchs in Deutschland“ (Bundesagentur für Arbeit 2009) haben die verschiedenen am Ausbildungsprozess in Deutschland beteiligten Akteure erstmals eine gemeinsame Definition formuliert. Demnach ist Ausbildungsreife zunächst grundsätzlich zu trennen von Berufseignung und Vermittelbarkeit. Zudem wurde in diesem Kriterienkatalog festgelegt, dass Ausbildungsreife ein mehrdimensionales Konzept ist, welches nicht nur kognitive Fähigkeiten sondern ebenfalls soziale und motivationale Dispositionen (z. B. Arbeitsverhalten, Durchhaltevermögen, Zuverlässigkeit, Lernbereitschaft und Höflichkeit) sowie die physische und psychische Eignung (z. B. altersgerechter Entwicklungsstand) umfassen sollte. Um neben der Bedeutung kognitiver Fähigkeiten auch dem Einfluss von motivationalen und Persönlichkeitsaspekten gerecht zu werden, betrachten wir in unseren Analysen zusätzlich auch die Selbstwirksamkeitserwartung der Jugendlichen. Die Selbstwirksamkeitserwartung wurde ebenfalls in der TREE-Studie erfasst und gerade für leistungsschwache Jugendliche könnte eine positive Selbstwirksamkeit (als Indikator für Ausdauer und Anstrengung der Persönlichkeit) ein wichtiger Schlüssel für den erfolgreichen Übergang in eine reguläre Ausbildungsstelle sein (vgl. auch Solga et al. 2012).

Auch wenn zur Kennzeichnung von Ausbildungsreife auch nicht-kognitive Fähigkeiten relevant sind, konzentrieren wir uns in unseren nachfolgenden Analysen bewusst auf den Einfluss kognitiver Fähigkeiten und greifen somit bei unserer „Definition“ von Ausbildungsreife auf das kognitive Kompetenzniveau der Jugendlichen zurück. Denn in Deutschland wird gerade von Arbeitgeberseite argumentiert, dass zunehmend nachlassende kognitive Fähigkeiten für die Verschlechterung der beruflichen Ausbildungschancen verantwortlich sind (siehe z.B. AG Mittelstand 2009; DIHK 2009; für einen zusammenfassenden Überblick siehe Eberhard 2006). Laut Wirtschaftsvertretern soll es einen relativ hohen Prozentsatz von Jugendlichen geben, deren kognitive Fähigkeiten so gering sind, dass sie schlichtweg nicht für eine berufliche Ausbildung geeignet sind. So warnte die AG Mittelstand in einem im November 2009 veröffentlichten Positionspapier davor, dass die in der PISA-Studie aufgedeckten geringen Kompetenzen unter Jugendlichen in Deutschland ein großes Problem für Ausbildungsbetriebe sind: „Für diese [kompetenzschwachen] Jugendlichen wird es sehr schwer, einen Ausbildungsplatz zu finden [...]“ (AG Mittelstand 2009, S. 2). Ob Jugendliche mit geringen kognitiven Kompetenzen tatsächlich ungeeignet sind für den Beginn einer Ausbildung, werden wir für das Nachbarland Schweiz empirisch untersuchen. 
Konkret haben wir für unsere Analysen folgende Arbeitshypothesen:

1. Die (Aus-) Bildungswege nach Ende der Schulpflicht werden sich für kompetenzschwache Jugendliche deutlich von denen kompetenzstärkerer Jugendliche unterscheiden. Aufgrund ihrer höheren kognitiven Kompetenzen (und damit verbunden höheren schulischen und akademischen Erfolgsaussichten; vgl. auch Breen und Goldthorpe 1997; Esser 1999) werden sich leistungsstarke Jugendliche viel häufiger für die Fortsetzung ihrer schulischen Laufbahn entscheiden als Jugendliche mit niedrigen kognitiven Kompetenzen. Leistungsschwache Jugendliche werden dagegen eher aus dem allgemeinbildenden Schulsystem ausscheiden, um sich auf die Suche nach einer beruflichen Ausbildung zu machen.

2. Anders als in Deutschland erwarten wir für die Schweiz jedoch, dass auch kompetenzschwache Jugendliche gute Einstiegschancen in eine reguläre berufliche Ausbildung haben und sich nach Ende der Schulpflicht nicht primär in Überbrückungsmaßnahmen (welche vergleichbar sind mit dem deutschen Übergangssystem) wiederfinden.

3. Die strengeren formalen Auswahlprozesse in größeren Betrieben sollten sich negativ auf die Berufsausbildungschancen von Jugendlichen mit geringen Kompetenzen auswirken. Deswegen erwarten wir, dass ein höherer Anteil von Klein- und Mikrobetrieben in der Region die Chancen von leistungsschwachen Jugendlichen verbessert, nach Ende der Schulpflicht eine reguläre Berufsausbildung aufzunehmen.

4. Wir erwarten, dass gerade für kompetenzschwache Jugendliche eine positive Selbstwirksamkeitserwartung ein wichtiger Faktor für den erfolgreichen Übergang in eine berufliche Ausbildung nach Ende der Schulpflicht ist, da sie damit die mit unzureichenden kognitiven Kompetenzen verbundenen Übergangsprobleme kompensieren können. Eine hohe Selbstwirksamkeit sollte also die Übertrittschancen von leistungsschwachen Jugendlichen in eine Berufsbildung signifikant erhöhen.

Abschließend bleibt anzumerken, dass sich die in Deutschland geführte Diskussion der mangelnden Ausbildungsfähigkeit bestimmter Jugendlicher aufgrund mangelnder kognitiver Kompetenzen unterstützen ließe, wenn unsere Ergebnisse belegen, dass auch in der Schweiz eine berufliche Qualifizierung von kognitiv schwachen Jugendlichen nicht gelingt. Sollten unsere Analysen jedoch zeigen, dass der Schweiz eine berufliche Qualifizierung von kompetenzschwachen Jugendlichen gelingt, dann wird der in Deutschland vorherrschenden Diskussion um die mangelnde Ausbildungsreife von bestimmten Jugendlichen der Boden entzogen.

\section{Daten und Methoden}

Für unsere Analysen werden wir auf die schweizerische Studie TREE („Transitions from Education to Employment") zurückgreifen. TREE ist die erste nationale Längsschnittuntersuchung in der Schweiz zum Übergang Jugendlicher von der Schule ins Erwachsenenleben. Im Zentrum der Studie steht die Dokumentation der Ausbildungsund Erwerbsverläufe von Jugendlichen nach Austritt aus der obligatorischen Schule. Die Ausgangsstichprobe von TREE sind die gut 6000 Jugendlichen, die an der Schweizer PISA-Erhebung von 2000 teilgenommen haben (vgl. Sacchi 2008). Für die Basisstich- 
probe von TREE wurden davon all die Jugendlichen ausgewählt, die zum PISA-Befragungszeitpunkt eine in der Regel öffentliche Schule der Sekundarstufe I besuchten und zum Ende des Schuljahres 1999/2000 die obligatorische Schule verlassen haben. Anders als in den meisten anderen Ländern, die an PISA 2000 teilgenommen haben, wurde in der Schweiz mit dem TREE-Projekt somit der weitere Lebensverlauf dieser Jugendlichen in jährlichen Befragungen verfolgt. Die Stichprobe ist national und sprachregional repräsentativ (ebd.). In unseren Analysen werden wir auf die bisher vorliegenden editierten Daten der TREE-Jugendstudie zurückgreifen, nämlich die Wellen 2000 bis 2007. ${ }^{4}$ Mit diesen Wellen können wir verlässlich den Übergang nach Beendigung der Schulpflicht analysieren und Jugendliche mit geringen Kompetenzen mit anderen Jugendlichen vergleichen.

Wie bei Panelstudien üblich, waren wir bei der Datenaufbereitung mit Lücken konfrontiert, in denen Jugendliche nicht an der Befragung teilgenommen haben oder nicht erreicht wurden. Wir lassen Lücken von maximal zwei Befragungsausfällen zu. Sollte die Person im dritten Jahr wieder teilgenommen haben, wurden die Aktivitäten seit der letzten Befragung dieser Person von TREE nachträglich erhoben, sodass wir diese Lücke „,auffüllen“ konnten. Personen mit einer Befragungslücke von mehr als 2 Jahren zur letzten erfolgreich durchgeführten Befragung haben wir rechtszensiert, um mögliche Fehler durch Fehlerinnern auszuschließen. Insgesamt umfasst unser Sample für die nachfolgenden Analysen 5847 Personen.

Als Jugendliche mit geringen Kompetenzen haben wir Jugendliche klassifiziert, die auf einer insgesamt sechs Kompetenzstufen umfassenden Skala in der 2000er PISA-Befragung lediglich über eine niedrige Lesekompetenz verfügten, nämlich einer Kompetenzstufen von ,sehr niedrig“, „,niedrig“ und „,eher niedrig“. Wir haben uns dafür entschieden, auch Jugendliche mit ,eher niedrigen“ Lesekompetenzen in diese Gruppe einzuschließen. Zwar hat Stalder (2011b) gezeigt, dass diese Jugendlichen etwas bessere nachobligatorische Bildungschancen haben als Jugendliche mit nur rudimentären Lesekompetenzen (also mit „niedrigen“ und ,sehr niedrigen“ Kompetenzen). Jedoch konnte Stalder (2011b) auch nachweisen, dass die kritische Schwelle, die über (Aus-) Bildungschancen entscheidet, weniger zwischen den Stufen „niedrig“ und „eher niedrig“ liegt sondern vielmehr zwischen den Kompetenzstufen ,eher niedrig“ und „eher hoch“.5 Insgesamt haben wir

4 Die TREE-Stichprobe ist eine Teilstichprobe der ursprünglichen PISA-Stichprobe (für einen Überblick siehe Sacchi 2008). Trotz des mehrstufigen Ziehungsverfahrens des ursprünglichen PISA-Samples werden wir für unsere Analysen keine Mehrebenen-Modelle anwenden, da die Übergänge nach Ende der Schulpflicht sehr vielfältig verlaufen und stark individualisiert sind (und somit vom Schul- oder Klassenkontext eher unabhängig sind, anders als beispielsweise bei der Untersuchung von Lehrerempfehlungen). Somit behandeln wir die TREE-Daten in den hier vorgestellten Analysen als Individual-Daten. Um der unterschiedlichen Ziehungswahrscheinlichkeit der beobachteten Individuen Rechnung zu tragen, haben wir unsere im folgenden präsentierten Ergebnisse noch einmal abgesichert, indem wir zusätzlich Cox-Regressionen berechnet haben, in welchen wir die Samplingstruktur berücksichtigen. Diese zusätzlichen Analysen haben gezeigt, dass unsere Ergebnisse und die uns interessierenden Effekte stabil sind.

5 Zudem haben wir unsere Berechnungen zur Validierung unserer nachfolgend dargestellten Befunde noch einmal durchgeführt und dabei lediglich die Personen mit „sehr niedrigen“ oder „niedrigen“"Lesekompetenzen als leistungsschwach klassifiziert. Auch bei dieser deutlich engeren Erfassung leistungsschwacher Jugendlicher bestätigen sich die Befunden und Schlussfol- 
so in unseren Daten 1860 Jugendliche mit geringen Kompetenzen identifiziert. 3982 Jugendliche mit eher höheren und höheren Lesekompetenzen sind die Vergleichsgruppe. Für fünf Personen in unserem Sample sind keine Lesekompetenzen verfügbar. In unseren Modellen wird mit einer Dummy-Variable für diese wenigen Personen kontrolliert.

Zur längsschnittlichen Analyse von Übergängen nach Beendigung der Schulpflicht verwenden wir Methoden der Ereignisanalyse, nämlich das periodenspezifische Exponentialmodell mit mehreren Folgezuständen oder sogenannten ,competing risks“ (Blossfeld und Rohwer 2002). Die Episode für Übergänge nach Beendigung der Schulpflicht haben wir wie folgt definiert: Personen unseres Samples werden im Mai 2000 (also dem Ende der Schulpflicht) ,at risk“ gesetzt und die Beobachtungsepisode beginnt. Diese Episode endet bei einem erfolgreichen Übergang in einen der folgenden Zustände: 1) Aufnahme einer beruflichen Ausbildung (hierunter fallen ebenfalls die 18 Personen, die in unserem Sample nach Ende der Schulpflicht eine Anlehre oder Attestausbildung aufnehmen), 2) Übergang in sogenannte Überbrückungsmaßnahmen, welche in der Schweiz Jugendliche auffangen, denen die Aufnahme einer regulären beruflichen Ausbildung nicht gelungen ist (z.B. das Absolvieren eines freiwilligen berufsvorbereitenden 10 . Schuljahres oder einer Vorlehre) und 3) Übergang in eine schulische Allgemeinbildung (Gymnasien, teilqualifizierende Mittelschulen). ${ }^{6}$ Um untersuchen zu können, ob leistungsschwachen Jugendlichen vor allem „nur“ die Aufnahme einer Ausbildung in Berufen mit geringem Anforderungsniveau gelingt, unterscheiden wir in weiteren Analysen zwischen beruflichen Ausbildungen mit 1) niedrigem, 2) mittlerem und 3) hohem intellektuellen Anforderungsniveau. ${ }^{7}$

gerungen der nachfolgend dargestellten Analysen, in welchen wir auch Jugendliche mit „,eher niedrigen" Lesekompetenzen als leistungsschwach klassifizieren. Auch bei Jugendlichen mit sehr geringen oder geringen Lesekompetenzen gelingt also der Mehrheit der Übergang in eine berufliche Ausbildung mit mittlerem oder hohem Anforderungsniveau. Auch zeigt sich, dass gerade Mikro- und Kleinbetriebe für diese Jugendlichen sehr wichtig sind, um den Übergang in die berufliche Ausbildung erfolgreich zu meistern.

6 Weiterhin können Jugendliche in der Schweiz nach Beendigung der Schulpflicht auch in tertiäre Bildung übergehen. Hierbei handelt es sich zumeist um Übergänge in eine Ausbildung im Bereich Krankenpflege, die sie mit 18 Jahren beginnen konnten. Da nur 107 Personen in unserem Sample diesen Übergang machen und keine dieser Personen geringe Kompetenzen aufweist, verzichten wir in unseren multivariaten Analysen auf der Darstellung dieser Ergebnisse.

7 Beim beruflichen Anforderungsniveau nach Stalder (2011a) handelt es sich um ein mittels Experten-Rating ermitteltes Maß zur Bestimmung der intellektuellen Fähigkeiten, die für das erfolgreiche Durchlaufen einer Berufsausbildung auf der Sekundarstufe II als minimal nötig erachtet wird. Das Anforderungsniveau wurde aus den in den Curricula festgehaltenen Lerninhalten und Ausbildungszielen der Lehrberufe abgeleitet, gefasst als eigenständige Größe unabhängig von anderweitigen beruflich erforderlichen Fähigkeiten und handwerklichen, sozialen o.ä. Fertigkeiten. Unter Berufen mit geringen intellektuellen Anforderungen finden sich beispielsweise Berufe wie Frisör/-in, Verkäufer/-in, Maler/-in, Maurer/in, Schneider/in oder Straßenbauer/-in. Berufe mit mittleren intellektuellen Anforderungen sind z. B. Dentalassistent/-in, Drucktechnologe/-in, Medizinische/r Praxisassistent/-in, Schreiner/-in, Elektromonteur/-in oder Fotograf/-in. Zu den Berufsausbildungen mit hohen intellektuellen Anforderungen gehö- 
Folgende Kovariaten lassen wir in unsere Analysen einfließen: 1) Geschlecht, 2) Migrationshintergrund (definiert, ob der Vater aus a) der Schweiz, b) Deutschland, Österreich, Frankreich oder Belgien, c) Italien oder Spanien, d) dem ehemaligen Jugoslawien, Portugal oder der Türkei oder e) einem anderen Land kommt), 3) soziale Herkunft des Jugendlichen (erfasst über den höchsten beruflichen Status (ISEI) der Eltern), 4) Region (deutsche Schweiz, französische Schweiz, italienische Schweiz), 5) Wohnort (urbanes vs. ländliches Gebiet, wobei Gemeinden ab 10.000 Einwohnern als urbaner Kontext gelten), 6) Schultyp (a) Sekundarschulen mit Grundanforderungen, b) Sekundarschulen mit erweiterten Anforderungen, c) Sekundarschulen mit hohen Anforderungen oder Gymnasien, d) Sekundarschulen ohne Selektion entsprechend den deutschen Gesamtschulen) und 7) Mathematiknote. ${ }^{8}$ Um die Ausbildungsreife der Jugendlichen abzubilden, nutzen wir 8) die Lesekompetenz als Maß für die kognitive Ausbildungsreife und 9) die Selbstwirksamkeitserwartung (beruhend auf dem gleichnamigen PISA-Index), um dem motivationalen Aspekt der Ausbildungsreife Rechnung zu tragen. Schließlich lassen wir 10) den Anteil von Mikro- und Kleinbetrieben an sämtlichen Ausbildungsbetrieben im Kanton (auf Basis von Daten der Schweizer Betriebszählungen 2001 und 2005) als Kovariate in unsere Modelle einfließen, um strukturelle Einflüsse des Ausbildungsmarktes auf die Ausbildungsschanzen junger Erwachsener zu überprüfen.

Die unter 1) bis 7) aufgelisteten unabhängigen Kovariaten werden in unseren Analysen vor allem als wichtige Kontrollvariablen dienen. Das Interesse unseres Beitrags liegt, wie oben ausgeführt, in der Erklärung der Ausbildungsfähigkeit von leistungsschwachen Jugendlichen. Somit konzentrieren wir uns in diesem Beitrag auf die Interpretation des Effekts des Kompetenzniveaus und seiner Interaktion mit dem Anteil von Mikro- und Kleinbetrieben sowie der Selbstwirksamkeitserwartung.

\section{Ergebnisse}

\section{1 (Aus-)Bildungsübergänge nach Beendigung der Schulpflicht}

Die Abbildungen 2 und 3 geben im ersten Schritt Aufschluss über die Dauer und die Art der Übergänge nach Beendigung der Schulpflicht von Jugendlichen mit niedrigen und eher hohen Lesekompetenzen. Wie Abb. 2 zeigt, gelingt beiden Gruppen der Übergang in eine weitere (Aus-) Bildung, sei es in eine berufliche Ausbildung, eine Überbrückungsmaßnahme oder die Fortführung der Schule, sehr schnell. Der Großteil der Jugendlichen setzt direkt nach der Sommerpause die (Aus-) Bildungskarriere fort. Leistungsschwache Jugendliche unterscheiden sich im Verlauf kaum von leistungsstärkeren Jugendlichen. Lediglich in den ersten 2 Monaten ist ein größerer Unterschied zwischen beiden Kurvenverläufen feststellbar und leistungsstärkere Jugendliche schaffen es etwas zügiger, den Übergang zu

ren Berufe wie Augenoptiker/-in, Bauzeichner/-in, Elektroniker/-in oder Informatiker/-in. In 311 der begonnen beruflichen Ausbildungen liegt uns das Anforderungsniveau nicht vor.

8 Es liegt die Information vor, ob die Mathematiknote am Ende der obligatorischen Schulzeit a) für das Bestehen des Faches ausreicht, b) über diesem Grenzwert liegt oder c) unter dem Grenzwert liegt. 


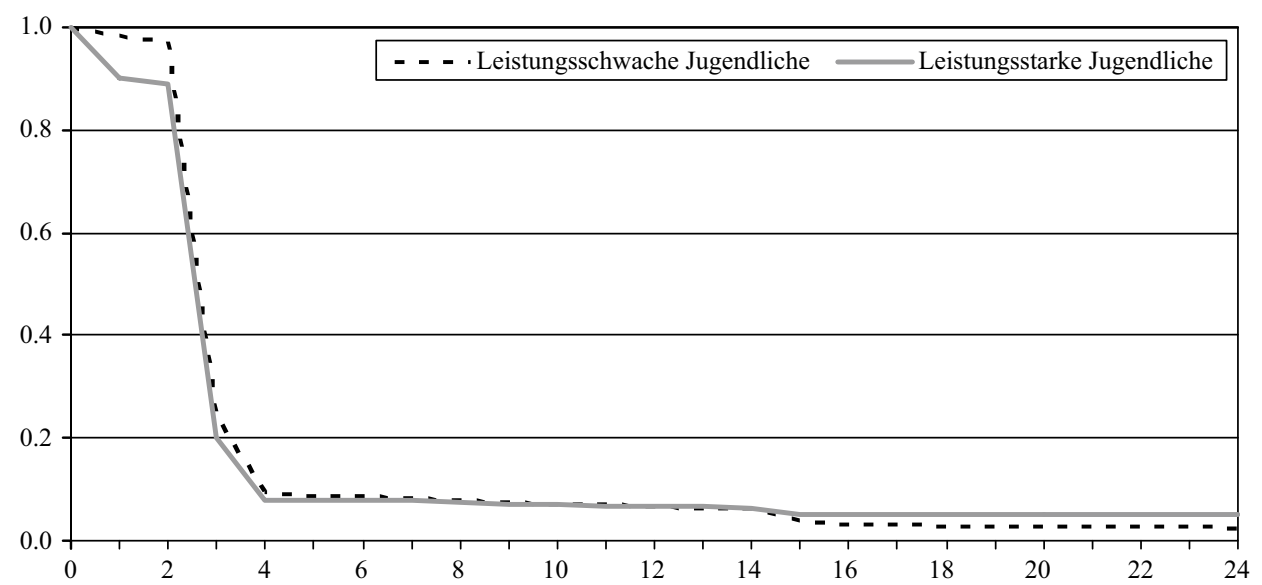

Abb. 2: Dauer (in Monaten) ohne berufliche Ausbildung, Überbrückungsmaßnahme oder weiterführende Schule nach Beendigung der Schulpflicht im Mai 2000 (Kaplan-Meier-Schätzung). (Eigene Berechnungen mit TREE-Daten)

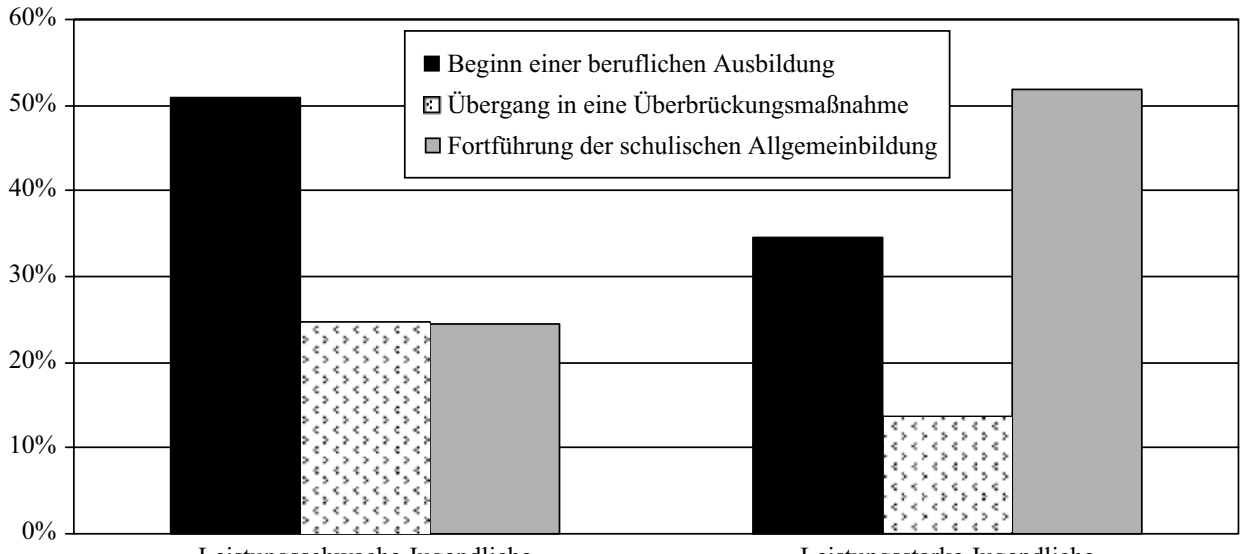

Leistungsschwache Jugendliche

Leistungsstarke Jugendliche

Abb. 3: Verteilung der Übergänge nach Beendigung der Schulpflicht auf verschiedene (Aus-) Bildungsangebote (in Prozent). (Eigene Berechnungen mit TREE-Daten)

machen. Dabei handelt es sich insbesondere um Übergänge in eine (zum Teil fortgesetzte) schulische Allgemeinbildung.

Deutliche Unterschiede zwischen den beiden Gruppen werden jedoch sichtbar, wenn die Art des Übergangs betrachtet wird (siehe Abb. 3). Wie zu sehen ist, setzen leistungsstärkere Jugendliche, wie erwartet, die schulische Ausbildung nach Beendigung der Schulpflicht sehr viel häufiger fort (knapp 50\%). Leistungsschwache Jugendliche scheiden dagegen viel häufiger aus der Schullaufbahn aus. Es gelingt dem Großteil der leistungsschwachen Jugendlichen in der Schweiz, nämlich 50\%, eine berufliche Ausbildung aufzunehmen. Zwar findet auch der Übergang in eine Überbrückungsmaßnahme unter 
leistungsschwachen Jugendlichen doppelt so häufig statt wie unter leistungsstärkeren Jugendlichen. Bemerkenswert ist jedoch mit Blick auf die deutsche Situation, dass auch unter den Jugendlichen mit geringen Kompetenzen der Übergang in eine reguläre Ausbildung sehr viel häufiger ist als ein Übergang in eine Überbrückungsmaßnahme.

Weitere, hier nicht abgebildete längsschnittliche Analysen haben zudem gezeigt, dass in der Schweiz die Suchzeit nach einer beruflichen Ausbildung mit einem Medianwert von 3,6 Monaten unter leistungsschwachen Jugendlichen im Vergleich zu Deutschland sehr gering ist. Diesen Jugendlichen gelingt somit ein direkter Übergang in eine berufliche Ausbildung nach Beendigung der Schulpflicht. Die Aufnahme einer Überbrückungsmaßnahme scheint dagegen, sowohl unter leistungsschwachen als auch leistungsstärkeren Jugendlichen, eine eher ungewünschte Notlösung zu sein, die erst nach vergeblicher und längerer Suche nach einer beruflichen Ausbildung gehäufter auftritt. Eine genauere Betrachtung der Art der Übergangsmaßnahme mit unseren Daten hat gezeigt, dass es sich bei diesen Maßnahmen vor allem um das Absolvieren eines freiwilligen berufsvorbereitenden 10. Schuljahres handelt (ca. 66\%). Die Jugendlichen, die kein freiwilliges berufsvorbereitendes 10. Schuljahr absolvieren, haben eine Vorlehre oder ein betriebliches Praktikum begonnen.

Erwähnenswert ist an dieser Stelle, dass in der Schweiz die Lage von Jugendlichen, die den Sprung in eine berufliche Ausbildung nicht geschafft haben, nicht hoffnungslos ist. Ganz im Gegenteil: Wie Hupka-Brunner et al. (2011) gezeigt haben, sind Überbrückungsmaßnahmen in der Schweiz insgesamt ein Sprungbrett. Dem Großteil dieser Jugendlichen gelingt nach Absolvieren einer Überbrückungsmaßnahme die Aufnahme einer beruflichen Ausbildung und anschließend auch der Einstieg in qualifizierte Erwerbsarbeit. Mit unseren Daten konnten wir dies durch weiterführende deskriptive Analysen bestätigen. So zeigt sich, dass fast $60 \%$ der Jugendlichen, die nach Ende der Schulpflicht nur den Übergang in eine Überbrückungsmaßnahme schaffen, bereits nach einem Jahr die Aufnahme einer beruflichen Ausbildung gelingt. Unterschiede für den Übergang in eine berufliche Ausbildung nach Teilnahme an einer Überbrückungsmaßnahme finden wir zwischen den leistungsschwachen und leistungsstarken Jugendlichen unseres Samples nicht. Nur $21 \%$ der leistungsschwachen Jugendlichen und 17\% der leistungsstarken Jugendlichen befinden sich länger (nämlich 2 oder mehr Jahre) in einer Überbrückungsmaßnahme, wobei auch hier wieder die Mehrheit bereits nach dem zweiten Jahr in einer Überbrückungsmaßnahme den Übergang in eine berufliche Ausbildung schafft. Diese weiterführenden Ergebnisse zeigen also, dass, wenn die Integration von leistungsschwachen Jugendlichen in eine berufliche Ausbildung in der Schweiz nicht schon direkt nach Ende der Schulpflicht gelingt, der Großteil der leistungsschwachen Jugendlichen diesen Übergang jedoch nach einem, spätestens 2 Jahren in einer Überbrückungsmaßnahme schafft. Überbrückungsmaßnahmen sind in der Schweiz somit tatsächlichen Brücken in den Beruf und keine Sackgassen, da die meisten Teilnehmer diese Programme als Sprungbrett in eine reguläre Berufsausbildung nutzen können (vgl. auch Hupka-Brunner et al. 2011; Imdorf 2011).

Eine große Schwäche der deutschen Ausbildungsstatistik ist, dass eine tatsächliche Evaluation und eine angemessene Analyse der Erfolgschancen von Programmen des Übergangssystems für Deutschland bisher nicht möglich ist (Beathge et al. 2007). Somit können wir keinen direkten Vergleich zwischen Deutschland und der Schweiz ziehen. 
Insgesamt scheint der Erfolg des deutschen Übergangssystems jedoch mehr als fraglich zu sein, insbesondere für Jugendliche ohne oder mit geringem Schulabschluss (Baethge et al. 2007; Baethge 2008; Gaupp und Reißig 2006; Reißig et al. 2008). Der Mehrheit der Jugendlichen im Übergangssystem gelingt nach Teilnahme kein Übergang in ein reguläres und qualifizierendes Ausbildungsverhältnis. Stattdessen sind viele von diesen Jugendlichen gefangen in einer Abfolge von Arbeitslosigkeit und weiteren Übergangsmaßnahmen (sogenannte Maßnahmenkarrieren). Dies gilt insbesondere für leistungsschwache Jugendliche, deren Situation meist hoffnungslos ist (Baethge et al. 2007; Baethge 2008; Gaupp und Reißig 2006).

Wie aber lassen sich diese guten Chancen von leistungsschwachen Jugendlichen bereits direkt nach Ende der Schulpflicht in der Schweiz erklären? Tabelle $2^{9}$ zeigt hierzu die Ergebnisse unserer multivariaten Analysen. Da das Interesse unseres Beitrags in der Erklärung der Ausbildungsfähigkeit von leistungsschwachen Jugendlichen liegt, konzentrieren wir uns hier auf die Interpretation der Effekte von Kovariaten zum Leistungsniveau. Die anderen Kovariaten fungieren als Kontrollvariablen in unseren Analysen. ${ }^{10}$ Wie schon in den vorangegangenen deskriptiven Befunden kann auch in diesen komplexeren Modellen und unter Kontrolle wichtiger Einflussfaktoren, wie soziale Herkunft, Migrationshintergrund oder Geschlecht, bestätigt werden, dass leistungsschwache Jugendliche eine signifikant höhere Neigung haben als leistungsstärkere Jugendliche, nach Beendigung der Schulpflicht in eine berufliche Ausbildung oder eine Überbrückungsmaßnahme überzugehen. Dagegen setzen leistungsstärkere Jugendliche bedeutend häufiger die Schullaufbahn fort.

Tatsächlich zeigt sich in Tab.2, dass die kantonale Ausbildungsbeteiligung von Mikro- und Kleinbetrieben einen wichtigen Einfluss für die Integrationschancen leistungsschwacher Jugendlicher hat (Modell 3 und 4). Je höher der Anteil von Mikro- und Kleinbetrieben, desto besser sind die Chancen von Jugendlichen mit geringen Kompetenzen, nach Ende der Schulpflicht eine reguläre berufliche Ausbildung aufzunehmen. Der ursprünglich signifikante Effekt für das Kompetenzniveau wechselt bei Hinzunehmen der Interaktion zwischen Kompetenzniveau und Anteil von Mikro- und Kleinbetrieben sogar die Richtung (Modell 4). Dies unterstreicht, wie wichtig in der Schweiz das Ausbildungsangebot durch Mikro- und Kleinbetriebe für leistungsschwache Jugendliche ist. Zwar gilt dies auch für die Aufnahme einer Überbrückungsmaßnahme. Wie jedoch das Modell 6 zeigt, ist der erfolgreiche Start ins Arbeitsleben durch die Aufnahme einer beruflichen Ausbildung (statt einer Überbrückungsmaßnahme) für leistungsschwache Jugendliche signifikant durch die Selbstwirksamkeitserwartung geprägt.

Überspitzt formuliert kann man die Ergebnisse also wie folgt zusammenfassen: Zwei Faktoren erklären in der Schweiz die guten Chancen von Jugendlichen mit geringen Kompetenzen, nach Beendigung der Schulpflicht erfolgreich in eine berufliche Ausbildung einzutreten, nämlich erstens und am deutlichsten durch das Vorhandensein von Ausbildungsplätzen in Mikro- und Kleinbetrieben, deren Auswahlverwahren bei Einstellungen

9 Tabelle 2 ist auf der Internetseite der KZfSS einsehbar (http://www.uni-koeln.de/kzfss/materialien/KS-64-4-buchholz.pdf).

10 Diese Kontrollvariablen für Geschlecht, Migrationshintergrund, soziale Herkunft, Region, Wohnort sowie Schultyp und Mathematiknote weisen die zu erwartende Richtung auf. 


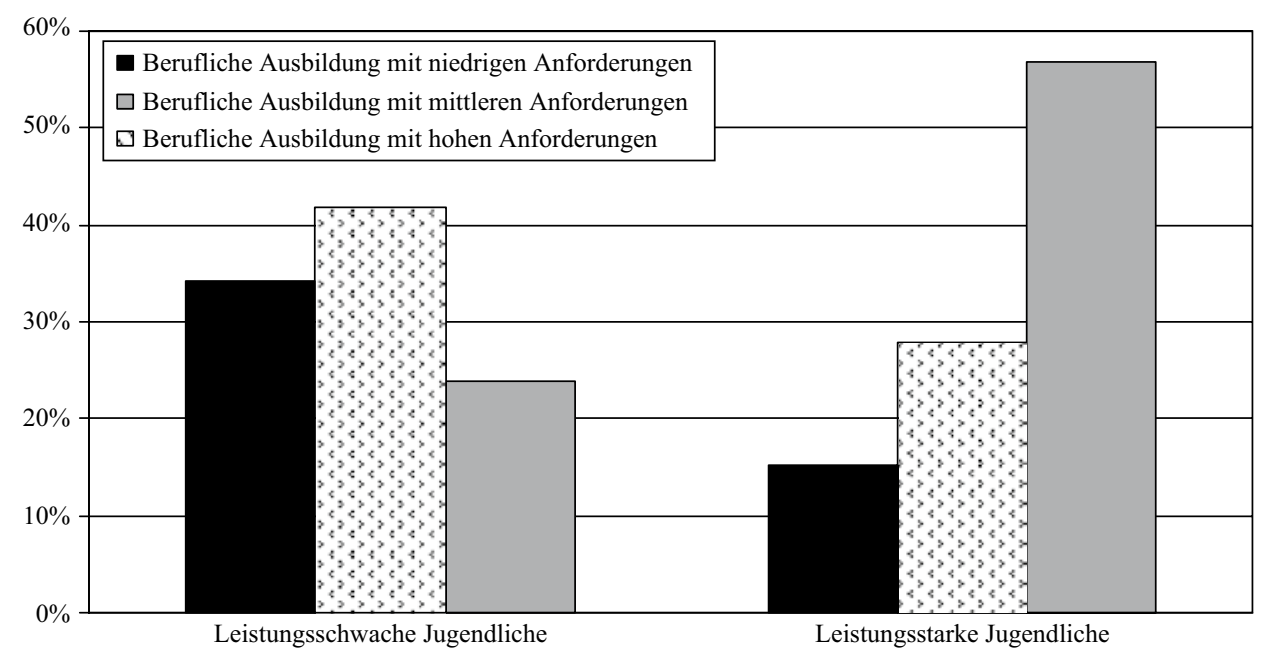

Abb. 4: Verteilung auf berufliche Ausbildungen mit niedrigen, mittleren und hohen Anforderungen (in Prozent). (Eigene Berechnungen mit TREE-Daten)

weniger selektiv sind als in Großbetrieben, und zweitens durch eine positive Selbstwirksamkeitserwartung, welche vor allem die Chancen für die Aufnahme einer regulären Ausbildung statt einer Überbrückungsmaßnahme beeinflusst.

\section{2 Übergänge in die berufliche Ausbildung nach Anforderungsniveau}

Im zweiten Schritt unserer Analysen betrachten wir nun zusätzlich das intellektuelle Anforderungsniveau der aufgenommenen beruflichen Ausbildung und unterscheiden im Folgenden zwischen beruflichen Ausbildungen mit niedrigem, mittlerem und hohem Anforderungsniveau. So wollen wir die Frage klären, ob die Integration von leistungsschwachen Jugendlichen in der Schweiz vielleicht vor allem durch das Aufnehmen von beruflichen Ausbildungen mit einem niedrigen intellektuellen Anforderungsniveau zu erklären ist.

Abbildung 4 gibt uns wieder einen ersten deskriptiven Überblick. In der Tat ist es so, dass leistungsschwache Jugendlich deutlich häufiger in eine Ausbildung mit niedrigen oder mittleren Anforderungen eintreten als leistungsstärkere Jugendliche, welchen vor allem die Aufnahme einer Ausbildung mit hohen Anforderungen gelingt. So haben knapp ein Drittel der Ausbildungen von leistungsschwachen Jugendlichen nur ein niedriges intellektuelles Anforderungsniveau, während dies lediglich für knapp 15\% der Ausbildungen der leistungsstärkeren Jugendlichen gilt. 42\% der leistungsschwachen Jugendlichen beginnen eine Ausbildung mit mittleren intellektuellen Anforderungen, im Vergleich zu $28 \%$ der leistungsstärkeren Jugendlichen. Der Mehrheit der leistungsstärkeren Jugendlichen (nämlich 57\%) gelingt die Aufnahme einer beruflichen Ausbildung mit hohen intellektuellen Anforderungen. Bei Jugendlichen mit geringen kognitiven Kompetenzen beläuft sich dieser Anteil auf knapp 24\%. Trotz dieser deutlichen Unterschiede zwischen den beiden Gruppen von Jugendlichen bleibt jedoch festzuhalten, dass 
in der Schweiz auch unter den leistungsschwachen Jugendlichen die Mehrheit (nämlich ca. 66\%) nicht auf einen Ausbildungsberuf mit niedrigen intellektuellen Anforderungen verwiesen wird. Im Gegenteil: Ausbildungen mit mittlerem Anforderungsniveau sind am häufigsten vertreten (mit knapp 42\%); fast einem Viertel der leistungsschwachen Jugendlichen gelingt sogar der Übergang in eine berufliche Ausbildungen mit hohen intellektuellen Anforderungen.

Weitere, hier nicht dargestellte längsschnittliche Analysen haben zudem gezeigt, dass die Suche nach einer Ausbildung mit niedrigen Anforderungen länger dauert. Der Median für einen Übergang in eine Ausbildung mit niedrigen Anforderungen liegt bei leistungsschwachen Jugendlichen bei knapp 14 Monaten, bei leistungsstärkeren Jugendlichen sogar bei fast 22 Monaten. Anscheinend versuchen Jugendliche beider Gruppen zunächst, eine Ausbildung mit mittleren oder hohen Anforderungen zu finden. Erst nach längerer (vergeblicher) Suche scheinen sie bereit zu sein, auch eine Ausbildung mit (nur) niedrigen intellektuellen Anforderungen zu akzeptieren. Jedoch unterscheiden sich auch hier leistungsstarke und leistungsschwache Jugendliche wieder deutlich. Für leistungsstärkere Jugendliche ist die Suchzeit für eine Ausbildung mit hohen intellektuellen Anforderungen am niedrigsten; der Median beträgt nicht einmal 3 Monate. Bei leistungsschwachen Jugendlichen ist die Suchzeit dagegen für Berufe mit mittleren intellektuellen Anforderungen am niedrigsten. Hier liegt der Median der Übergangszeit bei ungefähr 4 Monaten.

Wenden wir uns nun wieder den multivariaten Analysen zu, mit denen wir die Bestimmungsfaktoren für den Eintritt in eine berufliche Ausbildung mit niedrigen, mittleren oder hohen intellektuellen Anforderungen genauer identifizieren können (Tab. 3). ${ }^{11}$ Wir konzentrieren uns wieder auf die Interpretation der Effekte von Kovariaten, die Bezug auf das Leistungsniveau nehmen.

Zunächst wird auch in diesen multivariaten Analysen und unter Kontrolle weiterer Einflussfaktoren deutlich, dass Jugendliche mit geringen kognitiven Kompetenzen im Vergleich zu leistungsstärkeren Jugendlichen vor allem in Ausbildungen mit niedrigen und mittleren Anforderungen übergehen. Jugendliche mit höheren Kompetenzen beginnen dagegen signifikant häufiger eine Ausbildung mit hohem intellektuellem Anforderungsniveau. Zudem zeigt sich auch in unseren Ergebnissen zum Anforderungsniveau wieder die große Bedeutung der Mikro- und Kleinbetrieben für die Integrationschancen von leistungsschwachen Jugendlichen (Modelle 3 und 4). Für Jugendliche mit geringen Kompetenzen steigen die Chancen für die Aufnahme einer beruflichen Ausbildung mit mittleren oder hohen Anforderungen bedeutend, wenn der kantonale Ausbildungsmarkt stärker durch Mikro- und Kleinbetriebe geprägt ist (siehe Modelle 4 bis 6 für den Übergang in eine berufliche Ausbildung mit mittleren oder hohen Anforderungen). Dies unterstreicht noch einmal, wie wichtig das Vorhandensein von Mikro- und Kleinbetrieben für die Ausbildungschancen von leistungsschwachen Jugendlichen ist, und zwar nicht nur für ihre allgemeinen Ausbildungschancen sondern auch für das Anforderungsniveau der Ausbildung. ${ }^{12}$ Eine höhere Beteiligung von Klein- und Mikrobetrieben am kantonalen

11 Tabelle 3 ist auf der Internetseite der KZfSS einsehbar (http://www.uni-koeln.de/kzfss/materialien/KS-64-4-buchholz.pdf).

12 Es ist jedoch zu beachten, dass wir mit den von uns durchgeführten Analysen nicht klären, ob leistungsschwachen Jugendlichen diese Ausbildungen mit mittlerem und hohem Ausbildungs- 
Ausbildungsmarkt beeinflusst die Chancen, dass Jugendlichen mit niedrigen Kompetenzen eine Ausbildung mit mittlerem und hohem Anforderungsniveau aufnehmen, positiv. Dies gilt vor allem für den Übergang in Ausbildungen mit mittleren intellektuellen Anforderungen. Denn wie unsere Analysen zeigen, verschwindet der ursprünglich hoch signifikante Effekt für das Kompetenzniveau (Modelle 1 bis 3) sogar, sobald die Interaktion zwischen kantonalem Ausbildungsmarkt und Lesekompetenzen ins Modell einfließt (Modelle 4 bis 6).

Wie an den in Tab. 2 dargestellten Ergebnissen sichtbar ist, werden die Chancen leistungsschwacher Jugendlicher zur Aufnahme einer beruflichen Ausbildung (statt einer Überbrückungsmaßnahme) neben dem kantonalen Anteil von Mikro- und Kleinbetrieben auch durch eine positive Selbstwirksamkeitserwartung verbessert. Dieser Befund muss mit den hier in Tab. 3 dargestellten Ergebnissen nun so ergänzt werden, dass die besseren Ausbildungschancen von Jugendlichen mit positiver Selbstwirksamkeitserwartung vor allem damit zu erklären sind, dass diese eher bereit sind, eine Ausbildung mit niedrigen Anforderungen (statt einer Überbrückungsmaßnahme) zu beginnen.

\section{Zusammenfassung und Ausblick}

In Deutschland dominiert in der Diskussion um Ausbildungsplätze nun schon seit mehreren Jahren die These, dass ein Teil der Jugendlichen nach Ende der Schulpflicht nicht reif für eine berufliche Ausbildung ist. Gerade von der Wirtschaft wird die Position vertreten, dass es einen relativ hohen Prozentsatz von Jugendlichen geben soll, deren kognitive Kompetenzen so gering sind, dass sie nicht erfolgreich in die berufliche Ausbildung und den Arbeitsmarkt integriert werden können. Als Folge bleiben laut Vertretern der Wirtschaft viele Ausbildungsstellen unbesetzt (DIHK 2009). In Deutschland werden solche Jugendlichen auf das Übergangssystem verwiesen, dessen Bedeutung beträchtlich gestiegen ist und welches Teilnehmern keinen qualifizierenden Berufsabschluss vermittelt. Dies gilt insbesondere für schulisch schwache Jugendliche. So ist für Jugendliche mit und ohne Hauptschulabschluss in Deutschland der Zugang ins Übergangssystem heute eher die Regel und die Aufnahme einer regulären beruflichen Ausbildung eher die Ausnahme.

Ziel unseres Beitrages war es, die Integrationsfähigkeit von Jugendlichen mit geringen kognitiven Kompetenzen in eine berufliche Ausbildung zu untersuchen. Da für Deutsch-

niveau auch tatsächlich in Mikro- und Kleinbetrieben absolvieren, da es sich bei unserer Kovariate um ein Maß für die Unternehmensstruktur am kantonalen Ausbildungsmarkt handelt und nicht um eine Kovariate auf individueller Ebene der untersuchten Jugendlichen. Dass unsere Schlussfolgerung jedoch plausibel ist, zeigt sich auch anhand einer ungewichtete Kreuztabellierung unserer Daten für Betriebsgröße des ersten Ausbildungsbetriebs und Anforderungsniveau des ersten Ausbildungsberufs. Demnach werden in der Schweiz auch Ausbildungen mit mittlerem und hohem Anforderungsniveau mehrheitlich durch Mikro- und Kleinbetriebe angeboten. So verteilen sich Ausbildungen mit mittlerem Anforderungsniveau zu $82 \%$ auf Mikro- und Kleinbetriebe und nur zu 18\% auf Mittel- und Großbetriebe. Bei den Ausbildungen mit hohen intellektuellen Anforderungen entfallen immerhin noch $66 \%$ auf Klein- und Mikrobetriebe und $34 \%$ auf Mittel- und Großbetriebe. Bei den Ausbildungen mit niedrigen Anforderungen betragen die entsprechenden Anteile $86 \%$ vs. $14 \%$. 
land bisher keine Längsschnittdaten vorliegen, die es uns erlauben, den (Aus-) Bildungsverlauf von Jugendlichen mit geringen kognitiven Kompetenzen über mehrere Jahre zu verfolgen und mit leistungsstärkeren Jugendlichen zu vergleichen, nutzen wir für unsere empirischen Analysen Daten der TREE-Studie aus dem Nachbarland Schweiz. In der PISA-Studie 2000 war der Anteil von Schülern mit niedrigen und hohen Kompetenzen in beiden Ländern sehr ähnlich. Darüber hinaus ähnelt das schweizerische (Aus-) Bildungssystem dem Deutschen formal in vielen Punkten: So finden wir auch in der Schweiz ein eher stratifiziertes Schulsystem, das Jugendliche schon vergleichsweise früh in verschiedene Laufbahnen einteilt. Auch ist in der Schweiz wie in Deutschland vor allem die berufliche Ausbildung in einem dualen System dominierend. Ebenfalls ähnelt sich in beiden Ländern der institutionelle Umgang mit Jugendlichen, denen nicht direkt ein Übergang in eine reguläre Berufsausbildung gelingt. Trotz dieser formalen Ähnlichkeiten sind aber auch wichtige strukturelle Unterschiede zwischen beiden Ländern feststellbar, welche die Integrationschancen von leistungsschwachen Jugendlichen in der Schweiz deutlich verbessern dürften. So ist die allgemeine Arbeitsmarkt- und damit auch Ausbildungsmarktsituation in der Schweiz weitaus weniger angespannt, sodass schweizerische Arbeitgeber anders als in Deutschland viel stärker auch auf weniger ,attraktive“ potenzielle Arbeitnehmer und Auszubildenden angewiesen sind. Zudem finden wir in der Schweiz auf dem Ausbildungsmarkt eine weit größere Bedeutung von Mikro- und Kleinbetrieben, welche bei der Auswahl von Auszubildenden wahrscheinlich weniger selektiv sind als größere Unternehmen. Gerade wegen dieser Unterschiede ist die Schweiz ein spannender Testfall, um Licht in die seit Jahren in Deutschland herrschende Diskussion um eine nachlassende Ausbildungsreife von Jugendlichen zu werfen.

Wie unsere empirischen Ergebnisse zeigen, lässt sich die These, dass Jugendliche mit geringen Kompetenzen generell nicht ausbildungsfähig sind, für die Schweiz nicht bestätigen. Im Gegenteil: In der Schweiz gelingt der Mehrheit der kompetenzschwachen Jugendlichen ein reibungsloser und rascher Einstieg in eine berufliche Ausbildung. Und auch wenn der Anteil der leistungsschwachen Jugendlichen, die nach Ende der Schulpflicht vorerst in eine Überbrückungsmaßnahme übergehen, höher ist als unter leistungsstärkeren Jugendlichen, so ist dieser Anteil verglichen mit dem Anteil derer, die direkt in eine reguläre Ausbildung übergehen, relativ gering. Weiterführende Auswertungen haben zudem gezeigt, dass Überbrückungsmaßnahmen in der Schweiz auch tatsächlich Brücken und keine Sackgassen sind. Dem Großteil der Jugendlichen in der Schweiz, die direkt nach Ende der Schulpflicht nur den Übergang in eine Überbrückungsmaßnahme geschafft haben, gelingt bereits nach einem, spätestens nach zwei Jahren der Übergang in eine berufliche Ausbildung. Dies gilt im gleichen Maß für leistungsschwache wie auch leistungsstarke Jugendliche. Zwar zeigen unsere Analysen auch, dass sich leistungsschwache Jugendliche in der Schweiz häufiger als leistungsstarke Jugendliche in Ausbildungen mit nur niedrigen intellektuellen Anforderungen wiederfinden. Die Bedeutung von Ausbildungen in Berufen mit nur niedrigen Anforderungen darf jedoch nicht überschätzt werden. Denn betrachtet man die tatsächliche Verteilung von leistungsschwachen Jugendlichen auf Ausbildungen mit geringen, mittleren und hohen Anforderungen (Abb.4), so kann auch der Großteil der Jugendlichen mit geringen Kompetenzen, nämlich zwei Drittel, den Übergang in einen Ausbildungsberuf mit nur niedrigen intellektuellen Anforderungen vermeiden. Vor allem gelingt ihnen dies durch die Aufnahme einer Ausbildung mit 
mittleren Anforderungen, teilweise aber sogar durch die Aufnahme einer Ausbildung mit hohen intellektuellen Anforderungen.

Was aber ist das Erfolgsrezept der Schweiz? Unsere Ergebnisse bestätigen durchgängig, dass die Beteiligung von Mikro- und Kleinbetrieben am Ausbildungsmarkt die Ausbildungschancen von leistungsschwachen Jugendlichen signifikant verbessern. Unsere Erklärung hierfür ist, dass in Mikro- und Kleinbetrieben, anders als in größeren Betrieben, die in Deutschland den Ausbildungsmarkt viel stärker prägen als in der Schweiz, Kompetenz- und Leistungskriterien bei der Auswahl von Auszubildenden weit weniger Gewicht haben. Diese Betriebe sind offensichtlich viel eher bereit, auch leistungsschwachen Jugendlichen eine Chance auf eine berufliche Ausbildung zu geben. Eine Erklärung hierfür könnte zudem in der Organisation des Rekrutierungsprozesses liegen: Kurze Betriebspraktika noch während der Schulzeit sind in Schweizer Kleinbetrieben stark verbreitet. Auch für Deutschland ist bekannt, dass solche Praktika die Ausbildungschancen von Jugendlichen mit Hauptschulabschluss erhöhen können, da die Ausbilder so primär die persönlichen Stärken der Jugendlichen (und weniger ihre Defizite) kennenlernen (Solga et al. 2012).

Bemerkenswert ist, dass die Beteiligung von Mikro- und Kleinbetrieben am Ausbildungsmarkt nicht nur generell die Übergangschancen von leistungsschwachen Jugendlichen in eine berufliche Ausbildung bedeutend verbessern. Unsere empirischen Analysen zeigen, dass die Präsenz von Mikro- und Kleinbetrieben auch die Chancen erhöht, dass leistungsschwache Jugendliche eine Ausbildung in Berufen mit mittleren oder hohen Anforderungen beginnen können. Allerdings bleibt zu fragen, welche Auswirkung diese große Bedeutung von Mikro- und Kleinbetrieben für die Ausbildungschancen kompetenzschwacher Jugendliche auf die Ausbildungsqualität hat. So zeigt Ebbinghaus (2009), dass die Ausbildungsvielfalt in kleineren Betrieben niedriger ist und dort weit weniger Wert auf die Anforderungen an Ausbilder sowie die Standardisierung ihrer Kenntnisse gelegt wird. Auch wenn damit die Gefahr besteht, dass sich die große Bedeutung von Mikround Kleinbetrieben nicht nur positiv auf die Ausbildungschancen von Jugendlichen mit geringen Kompetenzen auswirkt, so ist eine etwas schlechtere Ausbildung jedoch immer noch besser als gar keine Ausbildung. Dies gilt insbesondere für Arbeitsmärkte, die durch eine große Bedeutung von Berufszertifikaten gekennzeichnet sind. Darüber hinaus kann man für die Schweiz zeigen, dass Berufe mit mittleren und hohen Anforderungen, die generell höhere Qualitätsanforderungen an die Ausbildung stellen, mehrheitlich in Kleinbetrieben ausgebildet werden.

Was kann aber nun für Deutschland aus den hier präsentierten Befunden für die Schweiz mitgenommen werden? Sicher lässt sich die Situation in der Schweiz nicht direkt mit der Lage in Deutschland vergleichen. Somit können unsere Erkenntnisse auf Basis der schweizerischen Daten auch nicht eins zu eins auf Deutschland übertragen werden. Was sich aber auf Grundlage unserer Befunde für die Schweiz festhalten lässt, ist, dass das in Deutschland häufig angeführte (Vor-) Urteil, dass Jugendliche mit geringen kognitiven Kompetenzen nicht reif für eine Ausbildung sind, schwer haltbar ist. In der Schweiz ist sowohl die Aufnahme einer regulären beruflichen Ausbildung als auch, wie Hupka-Brunner et al. (2011) und Stalder et al. (2008) in weiterführenden Analysen zeigen konnten, der erfolgreiche Abschluss einer Ausbildung ebenso für leistungsschwache Jugendliche möglich. Dies legt die Vermutung nahe, dass die in Deutschland vor allem 
von Seiten der Wirtschaft geführte Argumentation, dass nachlassende kognitive Fähigkeiten von Jugendlichen am Ende der Schulpflicht für den Rückgang an Ausbildungsplätzen und das Offenbleiben von potenziell verfügbaren Ausbildungsplätzen verantwortlich ist (Eberhard 2006), zumindest in Teilen interessengesteuert ist, um ein nicht ausreichendes Ausbildungsengagement zu legitimieren. Wie die Schweiz zeigt, sind geringe kognitive Kompetenzen kein absolutes Ausschlusskriterium für den Beginn (und das erfolgreiche Abschließen) einer Lehre. Geringe kognitive Kompetenzen von Jugendlichen scheinen in Deutschland somit vielmehr instrumentell und, durch Verdrängungseffekte, als ein relatives Ausschlusskriterium in Zeiten einer ungünstigen Situation von Angebot und Nachfrage genutzt zu werden.

Zudem wurde deutlich, dass neben kognitiven auch motivationale Aspekte für die Ausbildungsreife von Bedeutung sind (vgl. auch Solga et al. 2012): Jugendliche mit hohen Selbstwirksamkeitserwartungen scheinen durchaus in der Lage zu sein, ihre schlechteren Lesekompetenzen auszugleichen. Dies wirft die Frage auf, inwiefern gerade gefährdete Jugendliche im Übergang von der Schule in den Beruf gestützt werden können, um ihr positives Selbstbild und die damit verbundene Motivation nicht zu verlieren. Des Weiteren stellt sich die Frage, inwiefern in Deutschland die Ausbildungsbereitschaft von kleinen und mittleren Unternehmen gestärkt werden könnte, damit ausbildungsschwache Jugendliche bessere Chancen auf dem Lehrstellenmarkt haben. Die Förderung von Ausbildungsvereinen mit Integrationsauftrag, die es Kleinbetrieben vermehrt ermöglicht, sich an der Ausbildung zu beteiligen, ist hier eine Möglichkeit. Die Verbundsausbildung verteilt Ausbildungsrisiken auf mehrere Verbundsbetriebe und reduziert damit die Risiko-Aversion bei der Einstellung neuer Auszubildender aus Sicht der beteiligten Betriebe (Imdorf und Leemann 2012).

Denn gerade mit Blick auf die anstehende und nicht mehr aufzuhaltende demografische Alterung, die auch am Arbeitsmarkt nicht spurlos vorüber gehen wird, ist das systematische Brachlegen von Humankapitalressourcen in Deutschland höchst problematisch. Künftig wird die Zahl der Schulabgänger, die eine Ausbildung antreten können, stark schrumpfen. Deutschland muss sich somit arbeitsmarkt- und bildungspolitisch der Herausforderung stellen, die Humankapitalressourcen für den Arbeitsmarkt nachhaltiger und effizienter zu sichern und zwar durch die Integration aller potenziellen Arbeitskräfte, auch diejenigen mit geringeren Kompetenzen, denen bisher eine mangelnde Ausbildungsreife zugeschrieben wird.

\section{Literatur}

AG Mittelstand. 2009. Positionspapier zur Ausbildungsreife. Die Ausbildungsreife der Lehrstellenbewerber ist der Schlüssel für die Sicherung des Fachkräftenachwuchses der Wirtschaft. Berlin: AG Mittelstand.

Artelt, Cordula, Wolfgang Schneider und Ulrich Schiefele. 2002. Ländervergleich zur Lesekompetenz. In PISA 2000 - Die Länder der Bundesrepublik Deutschland im Vergleich, Hrsg. Deutsches PISA-Konsortium, 55-94. Opladen: Leske + Budrich.

Autorengruppe Bildungsberichterstattung. 2006. Bildung in Deutschland. Ein indikatorengestützter Bericht mit einer Analyse zu Bildung und Migration. Bielefeld: Bertelsmann. 
Autorengruppe Bildungsberichterstattung. 2008. Bildung in Deutschland 2008. Ein indikatorengestützter Bericht mit einer Analyse zu Übergängen im Anschluss an den Sekundarbereich I. Bielefeld: Bertelsmann.

Autorengruppe Bildungsberichterstattung. 2010. Bildung in Deutschland 2010. Ein indikatorengestützter Bericht mit einer Analyse zu Perspektiven des Bildungswesens im demografischen Wandel. Bielefeld: Bertelsmann.

Baethge, Martin. 2008. Das berufliche Bildungswesen in Deutschland am Beginn des 21. Jahrhunderts. In Das Bildungswesen in der Bundesrepublik Deutschland, Hrsg. Kai S. Cortina, 541-597. Hamburg: Rowohlt.

Baethge, Martin, Heike Solga und Markus Wieck. 2007. Berufsbildung im Umbruch. Signale eines überfälligen Aufbruchs. Berlin: Friedrich-Ebert-Stiftung.

BIBB. 2010. Datenreport zum Berufsbildungsbericht 2010. Informationen und Analysen zur Entwicklung der beruflichen Bildung. Bonn: Bundesinstitut für Berufsbildung.

BIBB. 2012. Auszubildende nach Wirtschaftsbereichen in Deutschland zwischen 1999 und 2007. http://www.bibb.de/dokumente/pdf/ausbildungsquote_wirtschatsbereiche_d_1999-2007.pdf (Zugegriffen: 21. Sept 2012).

Blossfeld, Hans-Peter. 1985. Bildungsexpansion und Berufschancen. Empirische Analysen zur Lage der Berufsanfänger in der Bundesrepublik. Frankfurt a. M.: Campus Verlag.

Blossfeld, Hans-Peter, und Götz Rohwer. 2002. Techniques of event history modeling. London: Lawrence Erlbaum Associates.

Blossfeld, Hans-Peter, und Reinhold Stockmann. 1998/1999. The German dual system in comparative perspective. International Journal of Sociology 28:3-28.

Breen, Richard, und John Goldthorpe. 1997. Explaining educational differentials. Towards a former rational action theory. Rationality and Society 9:275-205.

Brzinsky-Fay, Christian. 2007. Lost in transition? Labour market entry sequences of school leavers in Europe. European Sociological Review 23:409-422.

Bundesagentur für Arbeit. 2009. Nationaler Pakt für Ausbildung und Fachkräftenachwuchs in Deutschland. Kriterienkatalog zur Ausbildungsreife. Berlin: Bundesagentur für Arbeit.

Bundesamt für Statistik (BFS). 2008. Eidgenössische Betriebszählungen 1995, 1998, 2001, 2005. Sektoren 2 und 3. GEOSTAT-Datenbeschreibung. Neuenburg: Bundesamt für Statistik.

Bundesamt für Statistik (BFS) und Schweizerische Konferenz der kantonalen Erziehungsdirektoren (EDK). 2002. Für das Leben gerüstet? Die Grundkompetenzen der Jugendlichen - Nationaler Bericht der Erhebung PISA 2000. Bildungsmonitoring Schweiz. Neuchâtel: Bundesamt für Statistik \& Schweizerische Konferenz der kantonalen Erziehungsdirektoren.

Bundesministerium für Bildung und Forschung (BMBF). 2008. Berufsbildungsbericht 2008. Bielefeld: Bertelsmann.

Deutsche Industrie- und Handelskammer (DIHK). 2009. Ausbildung 2009. Berlin: DIHK.

Dietrich, Hans, Kathrin Dressel, Florian Janik und Wolfgang Ludwig-Mayerhofer. 2009. Ausbildung im dualen System und Maßnahmen der Berufsvorbereitung. In Handbuch Arbeitsmarkt 2009, Hrsg. Joachim Möller und Ulrich Walwei, 317-357. Bielefeld: Bertelsmann.

Ebbinghaus, Margit. 2009. Ideal und Realität betrieblicher Ausbildungsqualität - Sichtweisen ausbildender Betriebe. Bonn: Bundesinstitut für Berufsbildung.

Eberhard, Verena. 2006. Das Konzept der Ausbildungsreife - ein ungeklärtes Konstrukt im Spannungsfeld unterschiedlicher Interessen. Bonn: Bundesinstitut für Berufsbildung.

Esser, Hartmut. 1999. Soziologie. Spezielle Grundlagen. Bd.1: Situationslogik und Handeln. Frankfurt: Campus.

Gaupp, Nora, und Birgit Reißig. 2006. Welche Lotsenfunktionen sind wann für wen notwendig? Bildungswege benachteiligter Jugendlicher. In Übergangsmanagement. Jugendliche von der Schule ins Arbeitsleben lotsen, Hrsg. Tilly Lex, Nora Gaupp, Birgit Reißig und Hardy Adamczyk. München: Deutsches Jugendinstitut. 
Gaupp, Nora, Tilly Lex, Birgit Reißig und Frank Braun. 2008. Von der Hauptschule in Ausbildung und Erwerbsarbeit: Ergebnisse des DJI-Übergangspanels. Bonn: Bundesministerium für Bildung und Forschung.

Hupka-Brunner, Sandra, Nora Gaupp, Boris Geier, Tilly Lex und Barbara Stalder. 2011. Chancen bildungsbenachteiligter Jugendlicher: Bildungsverläufe in der Schweiz und in Deutschland. Zeitschrift für Soziologie der Erziehung und Sozialisation 31:62-78.

Imdorf, Christian. 2009. Die betriebliche Verwertung von Schulzeugnissen bei der Ausbildungsstellenvergabe. Empirische Pädagogik 23:392-409.

Imdorf, Christian. 2011. Zu jung oder zu alt für eine Lehre? Altersdiskriminierung bei der Ausbildungsplatzvergabe. Journal for Labour Market Research 45:79-98.

Imdorf, Christian, und Regula Julia Leemann. 2012. New models of apprenticeship and equal employment opportunity. Do training networks enhance fair hiring practices? Journal of Vocational Education and Training 64:57-74.

Kammermann, Marlise, und Armin Hättich. 2010. Mit Berufsattest in den Arbeitsmarkt. Ergebnisse einer schweizerischen Längsschnittstudie über die Berufsverläufe nach einer zweijährigen beruflichen Grundbildung. Berufsbildung in Wissenschaft und Praxis 5:11-14.

Moser, Urs. 2004. Jugendliche zwischen Schule und Berufsbildung. Eine Evaluation bei Schweizer Grossunternehmen unter Berücksichtigung des internationalen Schulleistungsvergleich PISA. Bern: h.e.p. Verlag.

Mühlemann, Samuel, Stefan Wolter, Marc Fuhrer und Adrian Wüest. 2007. Lehrlingsausbildung ökonomisch betrachtet. Ergebnisse der zweiten Kosten-Nutzen-Studie. Chur: Rüegger.

Müller, Barbara, und Jürg Schweri. 2012. Analysen zur Betriebszählung 2008. Die Betriebe in der dualen Berufsbildung - Entwicklungen 1985 bis 2008. Neuchâtel: Bundesamt für Statistik.

Müller, Walter, und Yossi Shavit. 1998. The institutional embeddedness of the stratification process. A comparative study of qualifications and occupations in thirteen countries. In From school to work. A comparative study of educational qualifications and occupational destinations, Hrsg. Yossi Shavit und Walter Müller, 1-48. Oxford: Clarendon.

Müller, Walter, Susanne Steinmann und Renate Ell. 1998. Education and labour-market entry in Germany. In From school to work. A comparative study of educational qualifications and occupational destinations, Hrsg. Yossi Shavit und Walter Müller, 143-188. Oxford: Clarendon.

Müller, Walter, Markus Gangl und Stefani Scherer. 2002. Übergangsstrukturen zwischen Bildung und Beschäftigung. In Bildung und Beruf. Ausbildung und berufsstruktureller Wandel in der Wissensgesellschaft, Hrsg. Matthias Wingens und Reinhold Sackmann, 39-64. Weinheim: Juventa.

Reißig, Birgit, Nora Gaupp und Tilly Lex. 2008. Hauptschüler auf dem Weg von der Schule in die Arbeitswelt. Übergänge in Arbeit. München: Deutsches Jugendinstitut.

Sacchi, Stefan. 2008. TREE-Langsschnittgewichtung: Konstruktion und Anwendung. Dokumentation zu den acht Erhebungswellen 2000 bis 2007. Bern: TREE \& cue sozialforschung.

Scherer, Stefani. 2005. Patterns of labour market entry - long wait or career instability? An empirical comparison of Italy, Great Britain and West Germany. European Sociological Review 22:1-14.

Schweizerische Koordinationsstelle für Bildungsforschung (SKBF). 2010. Bildungsbericht Schweiz. Aarau: Schweizerische Koordinationsstelle für Bildungsforschung.

Seibert, Holger, Sandra Hupka-Brunner und Christian Imdorf. 2009. Wie Ausbildungssysteme Chancen verteilen. Berufsausbildungschancen und ethnische Herkunft in Deutschland und der Schweiz unter Berücksichtigung des regionalen Verhältnisses von betrieblichen und schulischen Ausbildungen. Kölner Zeitschrift für Soziologie und Sozialpsychologie 61:595-620.

Sørensen, Aage B., und Arne L. Kalleberg. 1981. An outline of a theory of the matching of persons to jobs. In Sociological perspectives on labor markets, Hrsg. Ivar Berg, 49-74. New York: Academic. 
Stalder, Barbara. 2011a. Das intellektuelle Anforderungsniveau beruflicher Grundbildungen in der Schweiz. Ratings der Jahre 1999-2005. Basel: TREE Universität Basel.

Stalder, Barbara. 2011b. Berufsausbildung mit geringen Lesekompetenzen? Ergebnisse der Schweizer PISA-Folgestudie TREE. In Kompetenzdiagnostik in der beruflichen Bildung - Probleme und Perspektiven, Hrsg. Martin Fischer, Matthias Becker und Georg Spöttl, 190-203. Frankfurt a. M.: Peter Lang.

Stalder, Barbara, und Christof Nägele. 2011. Vocational education and training in Switzerland: Organisation, development and challenges for the future. In Transitionen im Jugendalter: Ergebnisse der Schweizer Längsschnittstudie TREE, Hrsg. Manfred M. Bergman, Sandra Hupka-Brunner, Anita Keller, Thomas Meyer und Barbara Stalder, 18-39. Zürich: Seismo.

Stalder, Barbara, Tilly Meyer und Sandra Hupka-Brunner. 2008. Leistungsschwach - Bildungsarm? Ergebnisse der TREE-Studie zu den PISA-Kompetenzen als Prädiktoren für Bildungschancen in der Sekundarstufe II. Die Deutsche Schule 100:436-448.

Thurow, Lester C. 1979. A job competition model. In Unemployment and inflation, Hrsg. Michael J. Piore, 17-32. New York: Sharpe.

Ulrich, Joachim G. 2008. Jugendliche im Übergangssystem - eine Bestandsaufnahme. Hamburg: Institut für Berufs- und Wirtschaftspädagogik.

Sandra Buchholz, 1976, Dr. rer. pol., vertritt den Lehrstuhl für Soziologie 1 an der Universität Otto-Friedrich-Universität Bamberg. Forschungsgebiete: Soziale Ungleichheiten; Soziologie des internationalen Vergleichs; Soziologie der Dynamik Sozialer Systeme und des sozialen Wandels; Statistik (insbesondere Anwendung moderner Längsschnittverfahren im Rahmen der Lebenslaufforschung); empirische Sozialforschung. Wichtige Veröffentlichungen: Die Flexibilisierung des Erwerbsverlaufs. Eine Analyse von Einstiegs- und Ausstiegsprozessen in Ost- und Westdeutschland. Wiesbaden 2008; Aging populations, globalization and the labor market: comparing late working life and retirement in modern societies. Cheltenham 2011 (hrsg. mit H.-P. Blossfeld, K. Kurz); Globalized labour markets and social inequality in Europe. Basingstoke, UK 2011 (hrsg. mit H.-P. Blossfeld, D. Hofäcker, K. Kolb); Young workers, globalization and the labor market. Comparing early working life in eleven countries. Cheltenham 2008 (hrsg. mit H.-P. Blossfeld, E. Bukodi, K. Kurz); Globalization, uncertainty and late careers in society. London 2006 (hrsg. mit H.-P. Blossfeld. D. Hofäcker).

Christian Imdorf, 1971, Dr. phil., Förderungsprofessur am Institut für Soziologie der Universität Basel. Forschungsgebiete: Übergang Schule - Beruf, Bildungssysteme und Geschlechtersegregation, Berufsausbildung. Veröffentlichungen: $\mathrm{Zu}$ jung oder $\mathrm{zu}$ alt für eine Lehre? Altersdiskriminierung bei der Ausbildungsplatzvergabe, Zeitschrift für Arbeitsmarktforschung, Online first: DOI 10.1007/s12651-011-0093-3, 2011; Ermöglicht die Flexibilisierung in der Berufsausbildung mehr Chancengerechtigkeit bei der Ausbildungsplatzvergabe? Fallstudie eines Schweizer Ausbildungsverbundes, (mit R.J. Leemann). Wiesbaden 2011; Die Diskriminierung „ausländischer“ Jugendlicher bei der Lehrlingsauswahl. In Diskriminierung. Grundlagen und Forschungsergebnisse, Hrsg. U. Hormel und A. Scherr. Wiesbaden 2010.

Sandra Hupka-Brunner, 1974, Dr. phil., Ko-Leitung 'TREE - Transition von der Erstausbildung ins Erwerbsleben', Institut für Soziologie, Universität Basel. Forschungsgebiete: Transition, Bildungsforschung, soziale Ungleichheit, Migration. Veröffentlichungen: Transitionen im Jugendalter: Ergebnisse der Schweizer Längsschnittstudie TREE, Zürich 2011 (mit M. Bergman, A. Keller, T. Meyer und B.E. Stalder); Chancen bildungsbenachteiligter Jugendlicher: Bildungsverläufe in der Schweiz und in Deutschland, Basel 2011 (mit N. Gaupp, B. Geier T. Lex und B.E. Stalder); Leistungsschwach - Bildungsarm? PISA-Kompetenzen als Prädiktoren für nachobligatorische Bildungschancen (mit B.E. Stalder und T. Meyer). Die Deutsche Schule 100, 2008. 
Hans-Peter Blossfeld, 1954, Dr. rer. pol., Dr. h.c., Prof. of Sociology am European University Institute in Florenz, Italien. Bis August 2012 Inhaber der Lehrstuhls für Soziologie 1 an der Otto-Friedrich Universität Bamberg, Geschäftsführender Direktor des Instituts für bildungswissenschaftliche Längsschnittforschung (INBIL) an der Universität Bamberg, Leiter des Nationalen Bildungspanels (NEPS) und Leiter des Staatsinstituts für Familienforschung an der Universität Bamberg. Forschungsgebiete: Bildungssoziologie, Allgemeine Soziologie; Sozialstrukturanalyse; Arbeitsmarktforschung, Familiensoziologie, Bevölkerungssoziologie; Globalisierungsforschung; Soziologie des internationalen Vergleichs; Soziologie der Dynamik sozialer Systeme und des sozialen Wandels; Soziologie der wirtschaftlichen und sozialen Entwicklung; Schichtungs- und Mobilitätsforschung; Statistik (insbesondere Anwendung moderner Längsschnittverfahren im Rahmen der Lebenslaufforschung); formale Modellbildung; Methoden der Datenerhebung; empirische Sozialforschung. Veröffentlichungen: 35 Bücher und über 230 Artikel. 